\title{
A Mobility Calculus with Local and Dependent Types
}

\author{
Mario Coppo ${ }^{1, \star}$, Federico Cozzi ${ }^{2, \star \star}$, Mariangiola Dezani-Ciancaglini ${ }^{1, \star \star \star}$, \\ Elio Giovannetti ${ }^{1, \dagger}$, and Rosario Pugliese ${ }^{3, \ddagger}$ \\ ${ }^{1}$ Dip. Informatica, Univ. di Torino, corso Svizzera 185, Torino, Italy \\ ${ }^{2}$ Dip. Scienze Matematiche e Informatiche, Univ. di Siena, \\ pian dei Mantellini 44, Siena, Italy \\ ${ }^{3}$ Dip. Sistemi e Informatica, Univ. di Firenze, \\ viale Morgagni 65, Firenze, Italy
}

Dedicated to Jan Willem Klop on the occasion of his 60th birthday.

\begin{abstract}
We introduce an ambient-based calculus that combines ambient mobility with process mobility, uses group names to collect ambients with homologous features, and exploits co-moves and runtime type checking to implement flexible policies for controlling process activities. Types rely on group names and, to support dynamicity, may depend on group variables. Policies can dynamically change also through installation of co-moves. The compliance with ambient policies can be checked locally to the ambients and requires no global assumptions. We prove that the type assignment system and the operational semantics of the calculus are 'sound', and define a sound and complete type inference algorithm which, when applied to terms whose type decorations only express the desired policies, computes the minimal type annotations required for their execution. As an application of our calculus, we present a couple of examples and linger on the setting up of policies for controlling the activities of the entities involved.
\end{abstract}

\section{Introduction}

The foundational research on distributed and mobile computing, driven by the technological advances of the last decades, has produced a number of theoretical models (for example 27/12]29/7/3, to cite just a few), which can be generally assimilated to some form of distributed process calculus or of 'ambient' calculus.

\footnotetext{
* Partially supported by EU within the FET - Global Computing initiative, project DART IST-2001-33477.

** Partially supported by EU within the project IHP 'Marie Curie DisCo' HPMTCT-2001-00290.

${ }^{\star \star \star}$ Partially supported by EU within the FET - Global Computing initiative, project MIKADO IST-2001-32222 and MURST Cofin'04 project McTafi.

† Partially supported by EU within the FET - Global Computing initiative, project DART IST-2001-33477.

‡ Partially supported by EU within the FET - Global Computing initiative, project MIKADO IST-2001-32222, and FP6-2004-IST-FET Proactive, project SENSORIA proposal contract number 016004 .
}

A. Middeldorp et al. (Eds.): Processes... (Klop Festschrift), LNCS 3838, pp. 404 4442005.

(C) Springer-Verlag Berlin Heidelberg 2005 
All such models rely on (often sophisticated) type systems to express and check behavioural properties concerning mobility, resource access, security, etc. In most of them, a system or component is represented by a term $P$ of a given calculus, a type $\mathrm{V}$ assigned to $P$ and an environment $\Sigma$. In the standard view, as is well-known, the term $P$ abstractly describes the implementation, its type $\mathrm{V}$ may express some behavioural properties, and the environment $\Sigma$ is a set of assumptions on the outside world. There is thus the notion of a global environment, whose corresponding concrete scenario is one where all the interacting parties are known in advance to each other, so that static checks performed before execution ensure the correctness of the whole system.

In particular, type systems for ambient calculi are usually based on the notion of a process/ambient type which describes the kind of communication and the kind of mobility actions a process can perform and, at the same time, the kind of movements and actions an ambient can make because of the activity of its internal processes. Every ambient name is assigned (by a global assumption or by a name restriction) a type which is simply the type of the processes it is allowed to contain. The basic typing rule for such systems is therefore some variant of the rule:

$$
\frac{\Sigma, m: \mathrm{V} \vdash P: \mathrm{V}}{\Sigma, m: \mathrm{V} \vdash m[P] \text { is well typed }}(\text { АмB })
$$

When dealing with computing in wide-area distributed and mobile systems, however, static verification is impractical both because of the huge amount of information to be checked and because typing information could be partial, inaccurate or missing. In such 'open' and dynamic systems no global environment can be assumed; on the contrary, there usually exist several different local and autonomous computational environments. Moreover, interaction may take place between parties whose respective properties are unknown or only partially known to each other. If stopping the execution for re-checking is to be avoided, every potentially dangerous component must dynamically carry with it sufficient behavioural information that can be checked at runtime by the other components interacting with it (see, e.g., the approach based on proof-carrying code [33).

To model these scenarios, we propose here an ambient-based calculus which combines ambient mobility with general process mobility (like $\mathbf{M}^{3}[15$ ) and where there are no global assumptions on ambient names, since there is no static ambient type. Every ambient name $m$ may be used to build an ambient $m[P]$ with any desired content $P$; on the other hand, process movements are constrained by the presence of co-moves and by runtime type checking. Indeed, following [22], we define an operational semantics with types which exploits types to authorize or block reductions but is simpler than a full-fledged typed operational semantics, because it only checks that types agree with process movements.

Types rely on group names, sort of 'family names' that group ambients with homologous features. Mobility properties and co-actions (entrance permissions) are expressed in terms of groups. The notion of a group, however, can be as fine-grained as necessary: in principle, each ambient can be in a distinct group. Dynamicity is enhanced by means of group-dependent types: types can contain 
variables which communication can instantiate to group names. In this way, during computation, processes can acquire knowledge of new group names that can be used either in movement actions or to let processes in.

Our first result shows that the type assignment system and the operational semantics of our calculus are 'sound'. This means that in any reduction sequence, ambient and process movements always comply with the constraints expressed by the types associated to the single ambients, and the types of messages exchanged in communications always match. This is done by first proving a property of subject reduction, namely that an ambient's 'policy' - expressed by its inner type - is preserved by reduction, and then by proving a property of type safety, i.e., that a process or ambient's behaviour complies, at each reduction step, with the policy of the enclosing ambient.

Since the terms of our calculus are quite heavily decorated with types, one would like to avoid writing all those types explicitly, and to let the system partially infer them à la ML: ideally, one would like to write them only for specifying, within an ambient, the rights granted to incoming mobile processes, without having to introduce any extra type annotation.

Also, safety suggests that an ambient, before letting in a process coming from an untrusted ambient, must typecheck it to ascertain that its actual behavioural type agrees with the one it declares, i.e., with the rights it requires (see [23]). More generally, it is useful to know which are, for ambient types, the minimal requirements that ensure the initial consistency of the system.

Both these issues require a type inference algorithm which, when applied to processes whose type decorations only express ambient-access policies, computes the minimal rights needed for running them. Such an algorithm is therefore the second result we present, along with the proofs of its soundness and completeness.

The rest of the paper is organized as follows. In Section 2 the different constructs of the calculus are explained by means of a simple example. In Section 3 the calculus' syntax, type system and operational semantics are formally presented, while in Section 4 the soundness results are stated and proved. Section 5 illustrates an application of our calculus to modelling a public transportation system and to controlling mobility of the entities involved (e.g., trains and passengers). In Section [6, a type inference algorithm is defined and its soundness and completeness are proved. Finally, in Section 7 we draw some short conclusions and touch upon directions for future work and comparisons with related work.

\section{An Explanatory Example: The Publisher}

The scenario envisaged in the example consists of a publisher PUB that publishes a number of electronic journals to which different institutions may subscribe. Two kinds of institutions are present: those that are fully trusted by the publisher and whose names are known to it, and all the others, whose names are not known in advance to the publisher and which are only partially trusted (in a sense that will be apparent below). The whole system is therefore the parallel composition (via the operator 'I') of institutions and publisher: 


$$
\begin{aligned}
& \left(\nu g_{\text {inst }}\right)\left(\nu g_{t d l_{1}}\right) \ldots\left(\nu g_{t d l_{m}}\right)\left(\nu g_{\text {ins }}\right) \\
& \quad\left(\operatorname{INST}_{1}|\ldots| \operatorname{INST}_{m}\left|\left(\nu g_{d l_{1}}\right) \operatorname{INS}_{1}\right| \ldots\left|\left(\nu g_{d l_{n}}\right) \mathrm{INS}_{n}\right| \mathrm{PUB}\right)
\end{aligned}
$$

where the $\operatorname{INST}_{i}$ (with $i=1, \ldots, m$ ) are the known trusted institutions and the INS $_{i}$ (with $i=1, \ldots, n$ ) are those only partially trusted.

Each ambient has a name and a group name: the association of an ambient with a group is not established by a static assumption but is done in the construction of the term. Ambient names and group names respectively act as the ambient's first name and family name; different ambients with homologous features are generally assigned the same group. For example, all the trusted institutions are assigned the group $g_{\text {inst }}$, while all the others belong to the group $g_{\text {ins }}$; the group $g_{t d l_{i}}$ (for $i=1, \ldots, m$ ) labels the download processes originated by the trusted institution $\operatorname{INST}_{i}$, while the group $g_{d l_{i}}($ for $i=1, \ldots, n$ ) labels the download processes originated by the untrusted institution $\operatorname{INS}_{i}$.

The standard construct $(\nu g) P$ declares that the name $g$ is known only to process $P$; thus the fact that the group of processes originated by an untrusted institution is not known in advance to the publisher has been modelled in (2.1) by putting the publisher out of the scope of its declaration.

Each of these top-level components is an ambient containing other ambients and processes: the publisher contains the journals and a manager process; an institution is an ambient that sends the publisher a subscription request and hosts a number of download processes; these in turn are represented by mobile ambients moving from their institutions to the publisher (where they access the subscribed journal) and back.

$$
\begin{aligned}
& \mathrm{PUB}=p u b: g_{\text {pub }}\left(\left\langle\varnothing,\left\{g_{\text {jrn }}\right\}\right\rangle, \operatorname{com}(\mathrm{amb}, \text { group })\right)\left[\mathrm{MGR}\left|\mathrm{JRN}_{1}\right| \ldots \mid \mathrm{JRN}_{n}\right] \\
& \mathrm{INST}_{i}=\text { inst }_{i}: g_{\text {inst }}\left(\left\langle\varnothing,\left\{g_{\text {pub }}\right\}\right\rangle, \mathrm{shh}\right)\left[\mathrm{REQ}_{i}^{k}\left|\mathrm{TDL}_{i}^{1}\right| \ldots \mid \mathrm{TDL}_{i}^{h}\right] \\
& \left.\mathrm{INS}_{i}=\text { ins }_{i}: g_{\text {ins }}\left(\| \varnothing,\left\{g_{\text {pub }}\right\}\right\rangle, \operatorname{shh}\right)\left[\mathrm{RQ}_{i}^{k}\left|\mathrm{DL}_{i}^{1}\right| \ldots \mid \mathrm{DL}_{i}^{l}\right]
\end{aligned}
$$

As anticipated in the Introduction, an ambient's properties are purely local and are not committed to global assumptions on the ambient's name or group. So the construct $\alpha: \gamma \mathrm{V}[P]$ also contains a type $\mathrm{V} \equiv(\langle\mathscr{C}, \mathscr{E}\rangle\rangle, \mathrm{T})$ which coincides with the type of its inner process $P$. This type consists of two components, the mobility type $\langle\langle\mathscr{C}, \mathscr{E}\rangle$ and the communication type $\mathrm{T}$, which are sets of group names: $\mathscr{C}$ is the set of ambient groups which $\alpha$ is allowed to cross (driven by $P$ ) and $\mathscr{E}$ is the set of ambient groups which processes sent by $\alpha$ (and then by $P$ ) are allowed to enter. On the other hand $\mathrm{T}$ specifies the type of the messages that can be communicated within $\alpha$ by input and output actions of $P$. It can be shh if no input and output action can be performed by $P$, or it can be of the form $\operatorname{com}(\mathrm{W})$, where $\mathrm{W}$ is an ambient name, a group name or a capability type. For instance, the ambient $p u b$ of group $g_{p u b}$ can cross no ambients, can send processes only to ambients of group $g_{j r n}$ and internally communicates pairs consisting each of an ambient name and a group name.

The ambient typing rule therefore informally becomes:

$$
\frac{\Sigma \vdash \alpha: \text { amb } \quad \Sigma \vdash \gamma: \text { group } \quad \Sigma \vdash P: \mathrm{V}}{\Sigma \vdash \alpha: \gamma \mathrm{V}[P]: \mathrm{V}^{\prime}}
$$


where $\mathrm{V}^{\prime}$ is any well-formed process type. As usual, the ambient's external process type $\mathrm{V}^{\prime}$, which may be any type, is not to be confused with its inner type $\mathrm{V}$. The global environment $\Sigma$ is used only for associating types to variables: they can range over different ambients and processes, so global assumptions on their types are unavoidable.

The whole computation starts with a request being sent by an institution (trusted or untrusted) to the publisher for subscribing to a journal $\mathrm{JRN}_{k}$ :

$$
\begin{aligned}
\mathrm{REQ}_{i}^{k} & \left.=\text { to } p u b: g_{p u b} \text { with }(\| \varnothing, \varnothing\rangle, \operatorname{com}(\text { amb, group })\right) \cdot\left\langle j r n_{k}, g_{t d l_{i}}\right\rangle \\
\text { or } \mathrm{RQ}_{i}^{k} & \left.=\text { to } p u b: g_{p u b} \text { with }(\| \varnothing, \varnothing\rangle, \operatorname{com}(\mathrm{amb}, \text { group })\right) .\left\langle j r n_{k}, g_{d l_{i}}\right\rangle
\end{aligned}
$$

The request is a simple mobile process that, by performing a to action, moves from the institution to the publisher, where it communicates the journal's name and the institution's 'signature', i.e., the group name of its download ambients. We remark that, although this example employs dyadic communication, the formal definition of the calculus (which will be presented in the next section), for the sake of simplicity, allows only monadic communication.

The construct to $m: \gamma$ with V. $P$ (introduced in a basic form in the calculus $\mathbf{M}^{3}$ ) denotes a process that sends its continuation $P$ to a sibling ambient named $m$ of group $\gamma$, where it will behave in conformity with the declared type V. Static type checking ensures that the type of $P$ actually equals its declared type V. Here, the request process $\mathrm{REQ}_{i}^{k}$ announces that, once reached the publisher, it will communicate a pair consisting of an ambient name and a group name, without performing any mobility action.

At runtime, a to action can fire only if the target ambient accepts the incoming process through the consumption of a suitable co-move of the form co $\gamma$ with $\mathrm{U}$, where $\gamma$ is the group of the expected incoming process and $\mathrm{U}$ a mobility type. The firing of the to-co transition is subject to the dynamic check that the communication type of the incoming process complies with the one of the entered ambient and that its mobility type is bounded by $\mathrm{U}$ (this notion is rendered by a suitable notion of subtyping). Static type checking ensures that $U$ is in turn compatible with the mobility type of the entered ambient. On the other hand, dynamic checking of type compatibility between the incoming process and the entered ambient is necessary since, in the absence of a global type environment for ambient or group names, the type properties of the entered ambient can be known only at runtime. Note that in this way it is possible to allow processes of different groups to enter a same ambient with different mobility rights. Checking the mobility type of a process going from one ambient to another is motivated by the fact that this may be extremely dangerous for the receiving ambient, since the process could take complete control of it.

In our example the control is performed by the manager, which is a process running within the publisher and dedicated to handling incoming requests:

$$
\begin{aligned}
& \text { MGR }=\quad ! \operatorname{cog} g_{\text {inst }} \text { with }\langle\varnothing, \varnothing\rangle .(x \text { :amb, } y \text { :group }) . \\
& \text { down } x: g_{j r n} \text { with }(\langle\varnothing,\{y\}\rangle, \text { shh) . !co } y \text { with }\langle\varnothing,\{y\}\rangle \\
& \mid \text { ! co } g_{\text {ins }} \text { with }\langle\varnothing, \varnothing\rangle \text {. ( } x \text { :amb, } y \text { :group). } \\
& \text { down } x: g_{j r n} \text { with ( }\langle\varnothing, \varnothing\rangle \text {, shh) . !co } y \text { with }\langle\varnothing, \varnothing\rangle
\end{aligned}
$$


The first action performed by the manager is the consumption of the co-move co $g_{\text {inst }}$ with $\langle\varnothing, \varnothing\rangle$, which authorizes a process to enter the publisher only if it cannot move the publisher nor send processes from it. As previously described, subscription requests satisfy such constraint.

The co-move is consumed in the action; the standard replication operator '!' is therefore used to provide authorization for an unlimited number of accesses. After accepting the request, the manager receives from it the journal's name and the institution's signature, goes down into the journal and finally leaves there a co-move that will grant the institution's reading processes the right to access the journal. Depending on whether the request comes from a known trusted institution or from an unknown one, the co-move deposited in the journal will be slightly different: in the second case it grants more restricted rights, which will compel processes from unknown institutions to adopt a more controlled behaviour.

The down primitive is a process mobility primitive analogous to the to: it sends the continuation process down from its ambient into a child ambient. The set of process mobility primitives is completed by the up action, which sends the continuation process to the parent ambient. For the sake of simplicity, there is only one kind of co-move which synchronizes with any of the three kinds of process movements (to, up and down).

The type expression in the co construct may contain a variable bound by an input abstraction. For example, in the case of a request from a fully trusted institution the co-move deposited by the manager in the journal contains the variable $y$ : the journal will thus accept any reading agent coming from a download ambient of group $y$ and going back as a continuation process to a $y$-ambient (i.e., to an ambient of the same group $y$ ). The actual value of $y$ is provided by the input operation performed by the manager prior to dropping the co-move at the journal.

Journals are simple ambients that communicate their contents any number of times:

$$
\begin{aligned}
& \operatorname{JRN}_{k}=j r n_{k}: g_{j r n}\left.\left(\left\langle\varnothing,\left\{g_{t d l_{1}}, \ldots, g_{t d l_{m}}\right\}\right\rangle\right), \operatorname{com}(\text { paper })\right)[ \\
&\left.\left.!\langle\text { paper }\rangle \mid ! \operatorname{co~} g_{p u b} \text { with }\left\langle\varnothing \varnothing,\left\{g_{t d l_{1}}, \ldots, g_{t d l_{m}}\right\}\right\rangle\right]\right]
\end{aligned}
$$

The co-move initially present in the journal enables it to receive from the publisher (through the manager) the co-move that in turn will authorize the journal to accept reader agents from members of an institution, if this has subscribed the journal.

As we have seen, the latter 'dynamic' co-move, in case of a trusted institution, will authorize the reader agent to go back to its originating $y$-ambient; the former 'static' co-move, to be able to accept the dynamic one, must therefore authorize the movement to any possible value of $y$, i.e., it must explicitly mention the signatures (i.e., the groups of download ambients) of all the trusted institutions.

Finally, a download process performing an access to a subscribed journal is a mobile ambient going out from its institution into the publisher, where it sends a reading agent to the journal; the agent, represented by a process, reads a paper within the journal and then goes back to the download mobile ambient, which 
in turn goes back to the institution. In the case of fully trusted institutions such behaviour can be implemented exactly as described. In particular, since the above list of actions has to be performed strictly in the given order, the ambient's main internal component will be a process consisting of a sequence of prefixes, in parallel with the co-move needed to eventually take in again the reading process:

$$
\begin{aligned}
& \left.\mathrm{TDL}_{i}^{j}=\left(\nu t d l_{i}^{j}\right) t d l_{i}^{j}: g_{t d l_{i}}\left(\left\langle\left\{g_{p u b}, g_{\text {inst }}\right\},\left\{g_{j r n}\right\}\right\rangle, \text { com(paper }\right)\right)[ \\
& \text { out inst } \text { in }_{i} g_{\text {inst }} \text {. in } p u b: g_{p u b} \text {. } \\
& \text { to } \left.\left.j r n_{k}: g_{j r n} \text { with ( } \| \varnothing,\left\{g_{t d l_{i}}\right\}\right\rangle, \operatorname{com}(\text { paper })\right) \text {. } \\
& \text { (x: paper). } \\
& \text { to } \left.t d l_{i}^{j}: g_{t d l_{i}} \text { with }\left(\|\left\{g_{p u b}, g_{\text {inst }}\right\}, \varnothing\right\rangle, \text { shh }\right) \text {. } \\
& \text { out pub: } g_{\text {pub }} \text {. in inst } \text { in }_{i} g_{\text {inst }} . !\left(\langle x\rangle \mid \operatorname{co} g_{\text {inst }} \text { with }\left\langle\varnothing,\left\{g_{\text {inst }}\right\}\right\rangle\right) \mid \\
& \text { !co } g_{j r n} \text { with }\left\langle\left\{\left\{g_{\text {pub }}, g_{\text {inst }}\right\}, \varnothing\right\rangle\right]
\end{aligned}
$$

The main process starts by driving the ambient out of the institution and into the publisher, by means of the usual out and in actions of ambient calculi; the only difference w.r.t. the standard primitives is that in our calculus an action takes as arguments not only an ambient name, but also a group name.

Once the download ambient is in the publisher, the main thread asks to be allowed to jump into the journal, by promising that it will not move the journal itself, will have a continuation coming back to an ambient of group $g_{t d l_{i}}$, and will perform an input/output of messages of type paper.

The journal accepts the reader process because it contains the appropriate co-move; then, the process reads the paper in the journal through an input operation and goes back to the download ambient, with the accompanying declaration that it will continue there by driving the ambient across publisher and institution boundaries; which in fact is what it performs in the second-last line of the definition, before finally making available the paper to other processes in the institution.

For the agent to be allowed to return from the journal after reading the paper, the download ambient must contain a suitable co-move, which is shown in the last line of the definition. Its with-component $\left\langle\left\{g_{p u b}, g_{\text {inst }}\right\}, \varnothing\right\rangle$ exactly matches the one of the returning process.

It is important to observe that an ambient's mobility type $\langle\mathscr{C}, \mathscr{E}\rangle$ is an upper bound of the mobilities of the parallel processes it contains and will contain, in the sense that the set $\mathscr{C}$ collects all the group names that are arguments of in or out actions or are found in the $\mathscr{C}$ components of co-actions, while the set $\mathscr{E}$ analogously collects the arguments of to, up or down actions and the members of the $\mathscr{E}$ components of co-actions. The inclusion of the with components of the comoves guarantees that all the possible future contents of the ambient are taken into account and that therefore the ambient's inner type will not be changed by reduction. This is formally expressed by the theorems relative to soundness, proved in Section 4 .

As we have seen, the ambient's inner communication type must be kept at runtime in the ambient construct, to allow the check of incoming processes; on 
the contrary, the ambient's mobility type is not strictly needed at runtime, since its $\mathscr{C}$ and $\mathscr{E}$ are supersets of the respective components of the co-actions, which are the ones that perform the checks w.r.t. mobility. Nevertheless, we have chosen to also include the mobility type in the ambient syntax, and thus to explicitly attach to each ambient its complete inner type $(\| \mathscr{C}, \mathscr{E}\rangle, \mathrm{T})$, in order to make the calculus more perspicuous and to facilitate the expression of the soundness property.

For example, in the $\mathrm{TDL}_{i}^{j}$ component, the ambient named $t d l_{i}^{j}$ (and of group $\left.g_{t d l_{i}}\right)$ is labelled with the type ( $\left\langle\left\{g_{p u b}, g_{i n s t}\right\},\left\{g_{j r n}\right\}\right\rangle$, , com(paper)), corresponding to the fact that the ambient goes across the boundaries of publisher and institutions, and sends a process to a journal.

The download originating from an unknown institution differs from the previous case because the reading process, once read the paper, is not allowed to directly go back from the journal to the download mobile ambient, whose group is not known to the publisher and therefore not mentioned in the authorization to exit the journal. To be able to return to the download ambient, the reader must turn itself into a mobile ambient - in the example, we have named such an ambient with the secret name box - which is then able to leave the journal without needing a permission. The ambient box acts as a sort of sandbox wherein the otherwise potentially harmful process may run safely, because ambients, differently from processes, in the absence of the open primitive cannot directly act on their surrounding ambients, nor perform input/output operations; they can therefore move around without need of any co-move authorization and runtime check.

Once out of the journal, the reader agent may then go back to the download ambient, which of course knows the family name (i.e., the group) of the sandbox ambient and can therefore authorize the move. The rest of the process is like in the case of known institutions.

$$
\begin{aligned}
& \left.\mathrm{DL}_{i}^{j}=\left(\nu d l_{i}^{j}\right) d l_{i}^{j}: g_{d l_{i}}\left(\left\langle\left\{g_{p u b}, g_{\text {ins }}\right\},\left\{g_{j r n}\right\}\right\rangle, \text { com(paper }\right)\right)[ \\
& \text { out } i n s_{i}: g_{\text {ins }} \text {. in pub: } g_{\text {pub }} \text {. } \\
& \text { to } \left.j r n_{k}: g_{j r n} \text { with }(\| \varnothing, \varnothing\rangle, \operatorname{com}(\text { paper })\right) \text {. } \\
& \text { (x: paper) } \\
& \text { ( } \left.\nu \text { box }) \text { box: } g_{b o x}\left(\|\left\{g_{j r n}\right\}\left\{g_{d l_{i}}\right\}\right\rangle, \text { shh }\right) \\
& \text { [out } j r n_{k}: g_{j r n} \text {. to } d l_{i}^{j}: g_{d l_{i}} \text { with }\left(\left\langle\left\{g_{p u b}, g_{i n s}\right\}, \varnothing\right\rangle,\right. \text { com(paper)). } \\
& \text { out } \left.p u b: g_{p u b} \text {. in } \text { ins }_{i}: g_{\text {inst }} . !\left(\langle x\rangle \mid \operatorname{co} g_{\text {ins }} \text { with }\left\langle\varnothing,\left\{g_{\text {ins }}\right\}\right\rangle\right)\right] \mid \\
& \text { !co } g_{b o x} \text { with }\left\langle\left\{\left\{g_{p u b}, g_{i n s}\right\}, \varnothing\right\rangle\right]
\end{aligned}
$$

A side effect of the above implementation is that at each download access a new box ambient is created and then left empty within the publisher. However, the name restriction immediately transforms such ambients into garbage, which can be collected by means of the equivalence ( $\nu$ box $)$ box: $g_{b o x}\left(\left\langle\left\{g_{j r n}\right\}\left\{g_{d l_{i}}\right\}\right\rangle\right)$, shh) $[0] \equiv 0$.

\section{The Calculus}

This section formally introduces the calculus. The syntax of the pre-terms of the language (where type constraints are ignored) is given in Fig. 1. Processes are 


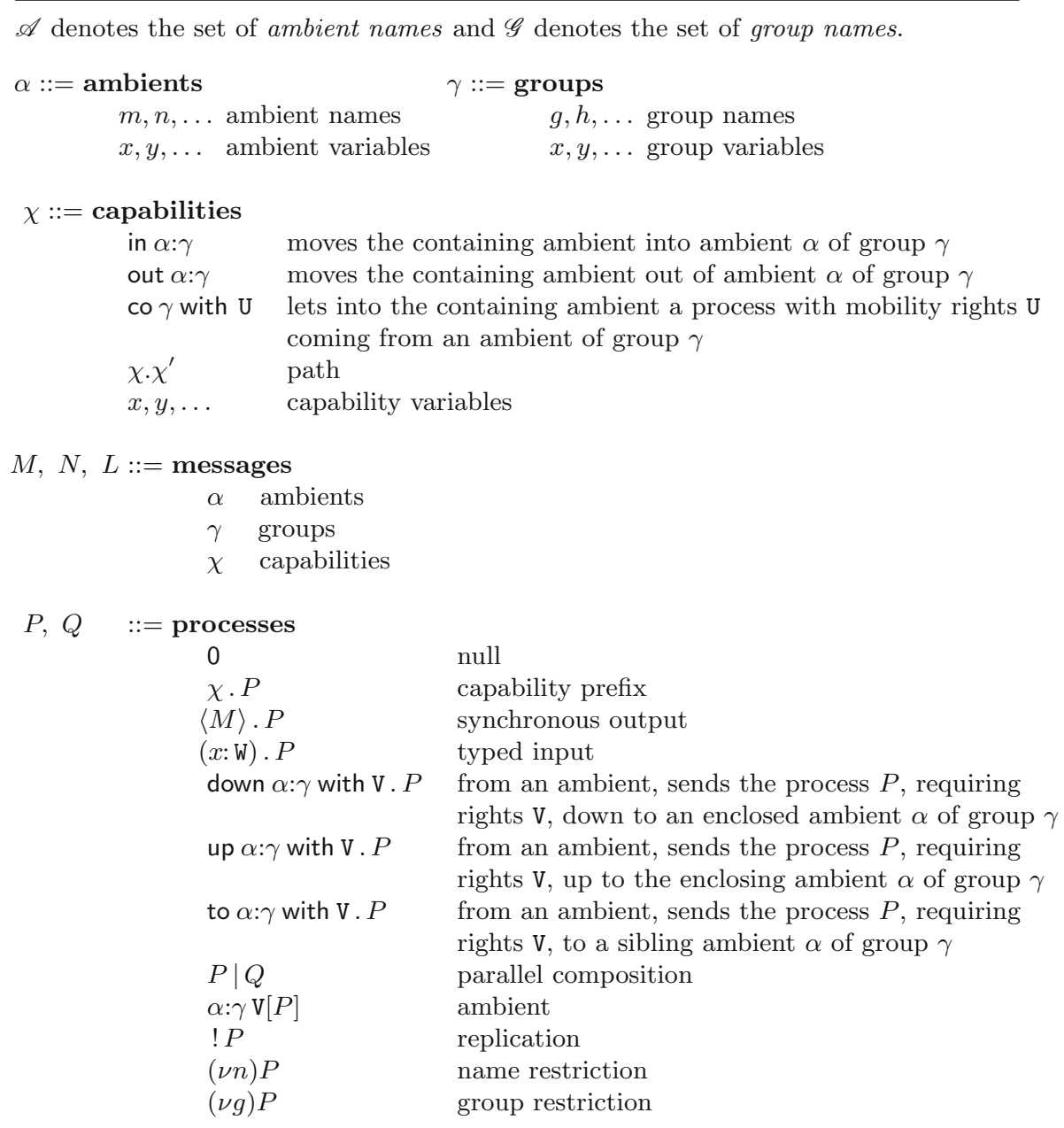

where U, V and W are defined in Figure 2.

Fig. 1. Syntax

built from the 0 process through the standard constructs of sequential prefixing, parallel composition, ambient formation, replication, name restriction and group restriction. Admissible syntactic prefixes are the capabilities in and out, the comove co, the input/output actions and the process movement constructs down, up and to. Messages can be ambient names, group names or (sequences of) capabilities; note that process movement actions (to, down, up) are not considered capabilities and cannot be communicated.

In the input construct $(x: \mathrm{W}) . P$ the prefix $(x: \mathrm{W})$ binds the variable $x$ in $P$, while in the name restriction $(\nu n) P$ the binder $(\nu n)$ binds the name $n$ in $P$; group re- 


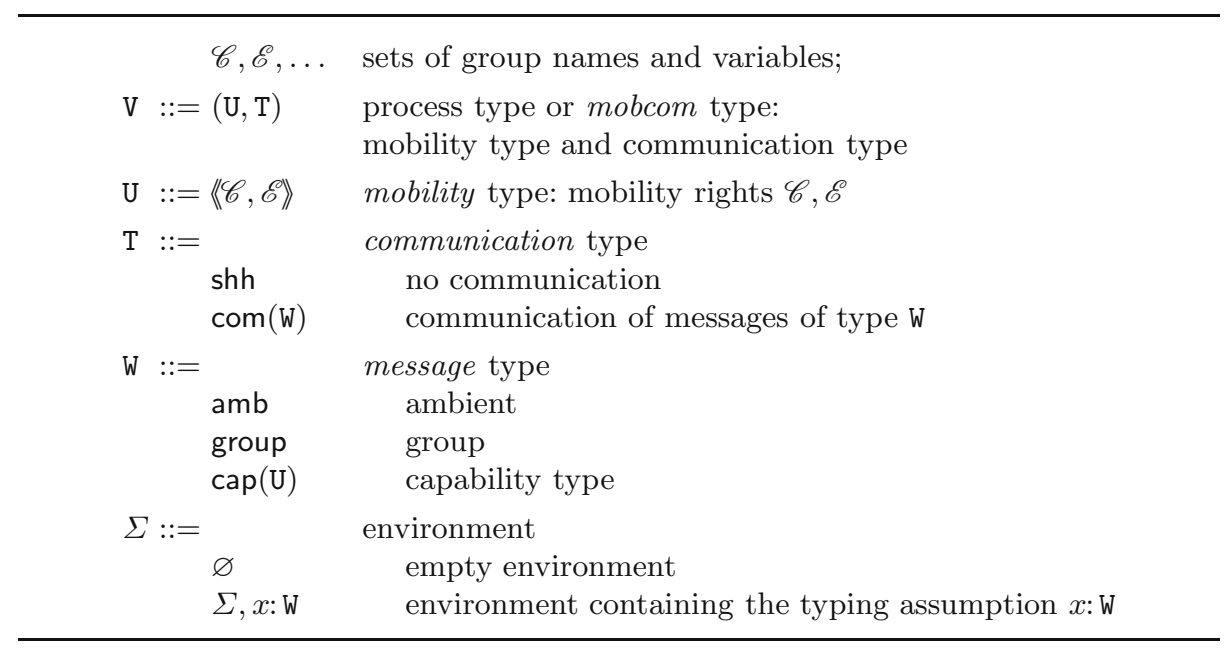

Fig. 2. Types

striction $(\nu g)$ works analogously. A name or a variable that is not bound is called free. The sets of free and bound names/variables of a term are respectively defined in accordance with that. We identify processes modulo renaming of bound names and variables. We will use the notation $\eta \in(\notin) \Upsilon$, where $\eta \in\{\alpha, \gamma\}$ and $\Upsilon \in\{P, \mathscr{C}, \mathrm{V}, \Sigma, \ldots\}$, as short for ' $\eta$ does (does not) occur free in $\Upsilon$ '.

The syntax of types is given in Fig. 2. Since types in our system describe communication and mobility properties, the type system is based on the notion of a mobcom type (or process type) $\mathrm{V}$ which packs the mobility type and the communication type of a process. A mobility type U consists of two sets $\mathscr{C}$ and $\mathscr{E}$ whose elements may be both group names and group variables; the intuitive meaning is that $\mathscr{C}$ is the set of the ambient groups which the enclosing ambient is allowed to cross when the process executes an in or out action, and $\mathscr{E}$ is the set of the ambient groups which the process may enter by means of a down, up or to action. As a matter of notation, if $\mathrm{V}=(\langle\mathscr{C}, \mathscr{E}\rangle, \mathrm{T})$, we will write $\mathscr{C}(\mathrm{V})$ and $\mathscr{E}(\mathrm{V})$ to denote $\mathscr{C}$ and $\mathscr{E}$, respectively; an analogous notation will be used for U.

Observe that types may depend on group variables, i.e. on parameters of input prefixes; they are therefore affected by reduction, when an input action substitutes a variable with a group name. Types, in turn, may occur in terms: mobcom types appear in the with components of the process mobility primitives (where they characterize process continuations) and in the ambient labels (where they represent the types of the inner processes); mobility types appear in the co-moves, where they represent the rights of the processes which are allowed to enter; message types appear in the input construct. The calculus is therefore a truly typed one, where types play an essential role both in the language definition and in the operational semantics.

The communication type $\mathrm{T}$ of a process indicates whether the process is silent (type shh) or can engage in the communication of messages of type W. A mes- 
sage type can be the atomic type group of group names, the atomic type amb of ambient names, or a capability type cap(U) representing the mobility rights required by capabilities.

A type environment $\Sigma$ is a finite set of pairs $x$ : W, where $x$ is a variable and W is its assumed message type. The domain $\operatorname{Dom}(\Sigma)$ of the environment $\Sigma$ is defined as usual:

$$
\operatorname{Dom}(\varnothing)=\varnothing \quad \operatorname{Dom}(\Sigma, x: \mathrm{W})=\operatorname{Dom}(\Sigma) \cup\{x\} .
$$

A subtyping relation $\leq$ is naturally defined on mobility types by componentwise set inclusion and then trivially extended to capability types, while the other two message types amb and group are not comparable with them. Subtyping on message types does not extend in the same way to communication types, since we do not allow subtyping polymorphism in input/output, except for the particular case of silent processes. Communication types are therefore not comparable with each other, with the exception of the type shh, which is the least element. We keep the same notation $(\leq)$ for both relations since there is no risk of confusion. Finally, subtyping on process (mobcom) types is the one trivially induced by subtyping on mobility and communication types.

\section{Definition 1 (Subtyping).}

1. $\left.\langle\mathscr{C}, \mathscr{E}\rangle \leq\left\langle\mathscr{C}^{\prime}, \mathscr{E}\right\rangle\right\rangle$ if $\mathscr{C} \subseteq \mathscr{C}^{\prime}$ and $\mathscr{E} \subseteq \mathscr{E}^{\prime}$

2. $\mathrm{amb} \leq \mathrm{amb} ;$ group $\leq$ group; $\operatorname{cap}(\mathrm{U}) \leq \operatorname{cap}\left(\mathrm{U}^{\prime}\right)$ if $\mathrm{U} \leq \mathrm{U}^{\prime}$;

3. $\mathrm{T} \leq \mathrm{T} ; \quad \operatorname{shh} \leq \mathrm{T}$;

4. $(\mathrm{U}, \mathrm{T}) \leq\left(\mathrm{U}^{\prime}, \mathrm{T}^{\prime}\right)$ if $\mathrm{U} \leq \mathrm{U}^{\prime}$ and $\mathrm{T} \leq \mathrm{T}^{\prime}$.

Whenever we write $\Sigma \subseteq \Sigma^{\prime}$, with $\Sigma$ and $\Sigma^{\prime}$ type environments, we mean the standard set-theoretic inclusion.

We now turn to the typing rules. There are seven kinds of typing judgments:

$$
\begin{aligned}
& \vdash \Sigma \\
& \Sigma \vdash \mathrm{U} \\
& \Sigma \vdash \mathrm{W} \\
& \Sigma \vdash \mathrm{T} \\
& \Sigma \vdash \mathrm{V} \\
& \Sigma \vdash M: \mathrm{W} \\
& \Sigma \vdash P: \mathrm{V}
\end{aligned}
$$

$$
\begin{aligned}
& \text { good environment } \Sigma \\
& \text { good mobility type } \mathrm{U} \\
& \text { good message type } \mathrm{W} \\
& \text { good communication type } \mathrm{T} \\
& \text { good mobcom type } \mathrm{V} \\
& \text { good message } M \text { of type } \mathrm{W} \\
& \text { good process } P \text { of type } \mathrm{V}
\end{aligned}
$$

In the sequel, the generic form $\Sigma \vdash \Gamma$ is intended to range over the last six kinds of judgments.

Figure 3 contains the rules for the well-formedness of environments and types, necessary because the calculus allows variables to occur within mobility types as long as they stand for group names. To simplify the presentation of the rules we rely on the established notational conventions to distinguish between the syntactic categories of objects. So, for instance, $W$ denotes a message type, $U$ a mobility type and so on. The rules are all standard; in particular, rule (MOB TYPE) checks that all the variables contained in a mobility type have been assumed to be of type group. 


$$
\begin{aligned}
& \frac{\forall \gamma \in \mathscr{C} \cup \mathscr{E} \quad \Sigma \vdash \gamma: \text { group }}{\Sigma \vdash\langle\mathscr{C}, \mathscr{E}\rangle} \quad \text { (MоB TYPE) } \\
& \frac{\vdash \Sigma}{\Sigma \vdash \text { group }} \text { (Group TyPe) } \frac{\vdash \Sigma}{\Sigma \vdash \text { amb }} \text { (AMB TyPe) } \frac{\Sigma \vdash \mathrm{U}}{\Sigma \vdash \operatorname{cap}(\mathrm{U})} \text { (CAP TyPe) } \\
& \frac{\vdash \Sigma}{\Sigma \vdash \operatorname{shh}}(\text { Shн Type }) \frac{\Sigma \vdash \mathrm{W}}{\Sigma \vdash \operatorname{com}(\mathrm{W})}(\text { MsgCom Type }) \frac{\Sigma \vdash \mathrm{U} \quad \Sigma \vdash \mathrm{T}}{\Sigma \vdash(\mathrm{U}, \mathrm{T})} \text { (MовCoм Type) } \\
& \overline{\vdash \varnothing}(\text { Empty Env }) \quad \frac{\Sigma \vdash \mathrm{W} \quad x \notin \operatorname{Dom}(\Sigma)}{\vdash \Sigma, x: \mathrm{W}} \text { (Env Constr) }
\end{aligned}
$$

Fig. 3. Good Types and Environments

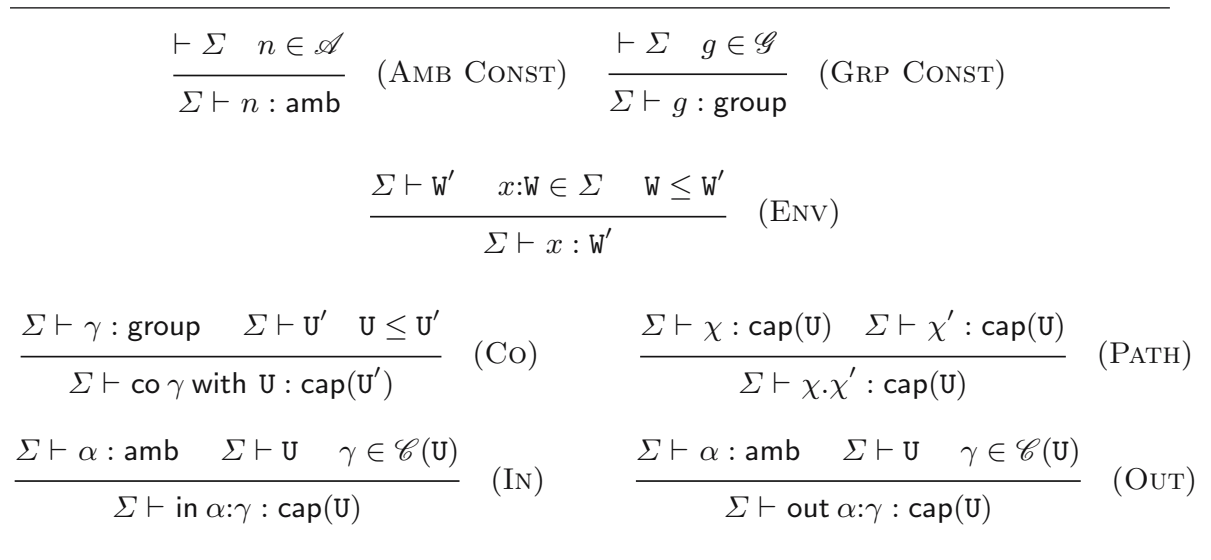

Fig. 4. Good Messages

The typing rules for messages and processes are given in Fig. 4 and Fig. 5 , respectively. Rules (AMB CONST) and (GRP CONST) are straightforward.

In the rule (ENV), if a variable is assigned a message type $W$ equal to the atomic type $a m b$ or group, its deduced type $W^{\prime}$ can only be the same as $W$. If, on the other hand, a variable is assumed to be of a capability type $\mathrm{W} \equiv \operatorname{cap}(\mathrm{U})$, then subsumption applies, and the deduced type $\mathrm{W}^{\prime}$ can be any supertype of $\mathrm{W}$.

As usual, subtyping enhances typability by allowing a subterm to have a type that is a subtype of the one required by the construction of the term. For example, the simple process $(x: \operatorname{cap}(\|\{g\}, \varnothing\rangle)) \cdot x$. in $m: g_{m} .0$ is well typed (in the empty environment), with typing:

$$
\left.\vdash(x: \operatorname{cap}(《\{g\}, \varnothing\rangle)) \cdot x \cdot \text { in } m: g_{m} \cdot 0:\left(《\left\{g, g_{m}\right\}, \varnothing\right\rangle, \operatorname{com}(\operatorname{cap}(\|\{g\}, \varnothing\|))\right)
$$




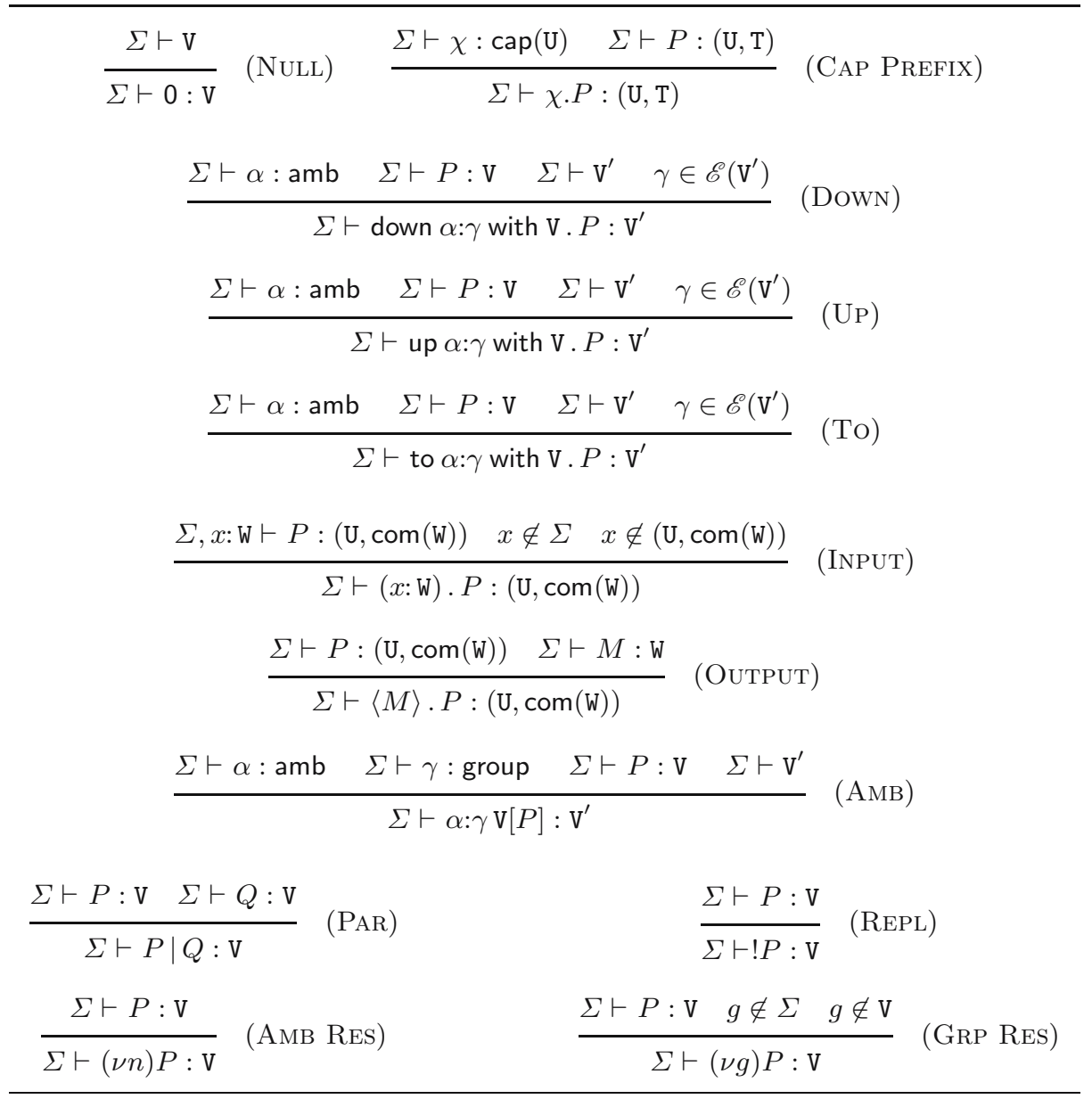

Fig. 5. Good Processes

where the capability that is going to be received in input carries a more restricted mobility than the resulting mobility of the whole process. Though quite natural, that would not be possible without subtyping.

Rule (Co) checks that the mobility type of the co-move is a supertype of the mobility type of the processes the co-move lets in. Rule (РATH) checks that the mobility types of the two (sequences of) capabilities are equal.

Rules (IN) and (OUT) check that the $\mathscr{C}$ component of the mobility type contains the group $\gamma$ of the ambient across whose border the capability drives its ambient; a similar check is performed in rules (Down), (UP) and (To) for the $\mathscr{E}$ component. Rule (AMB) ensures that the type of the inner process is recorded in the ambient header. The same rule allows an ambient to have an arbitrary type, as does rule (NULL) for the 0 process. 
Structural Congruence: $(\mid, 0)$ is a commutative monoid.

$$
\begin{aligned}
& (\nu n)(P \mid Q) \equiv(\nu n) P \mid Q \quad(n \notin Q) \quad(\nu n)(\nu m) P \equiv(\nu m)(\nu n) P \\
& n: g \mathrm{~V}[(\nu m) P] \equiv(\nu m) n: g \mathrm{~V}[P] \quad(n \neq m) \quad(\nu n)(\nu g) P \equiv(\nu g)(\nu n) P \\
& (\nu g)(P \mid Q) \equiv(\nu g) P \mid Q \quad(g \notin Q) \quad(\nu g)\left(\nu g^{\prime}\right) P \equiv(\nu g)\left(\nu g^{\prime}\right) P \\
& n: g \mathrm{~V}\left[\left(\nu g^{\prime}\right) P\right] \equiv\left(\nu g^{\prime}\right) n: g \mathrm{~V}[P] \quad\left(g \neq g^{\prime} \& g^{\prime} \notin \mathrm{V}\right) \quad ! P \equiv P \mid ! P \\
& (\nu n) 0 \equiv 0 \quad(\nu g) 0 \equiv 0 \quad(\nu n) n: g \mathrm{~V}[0] \equiv 0
\end{aligned}
$$

Basic reduction rules:

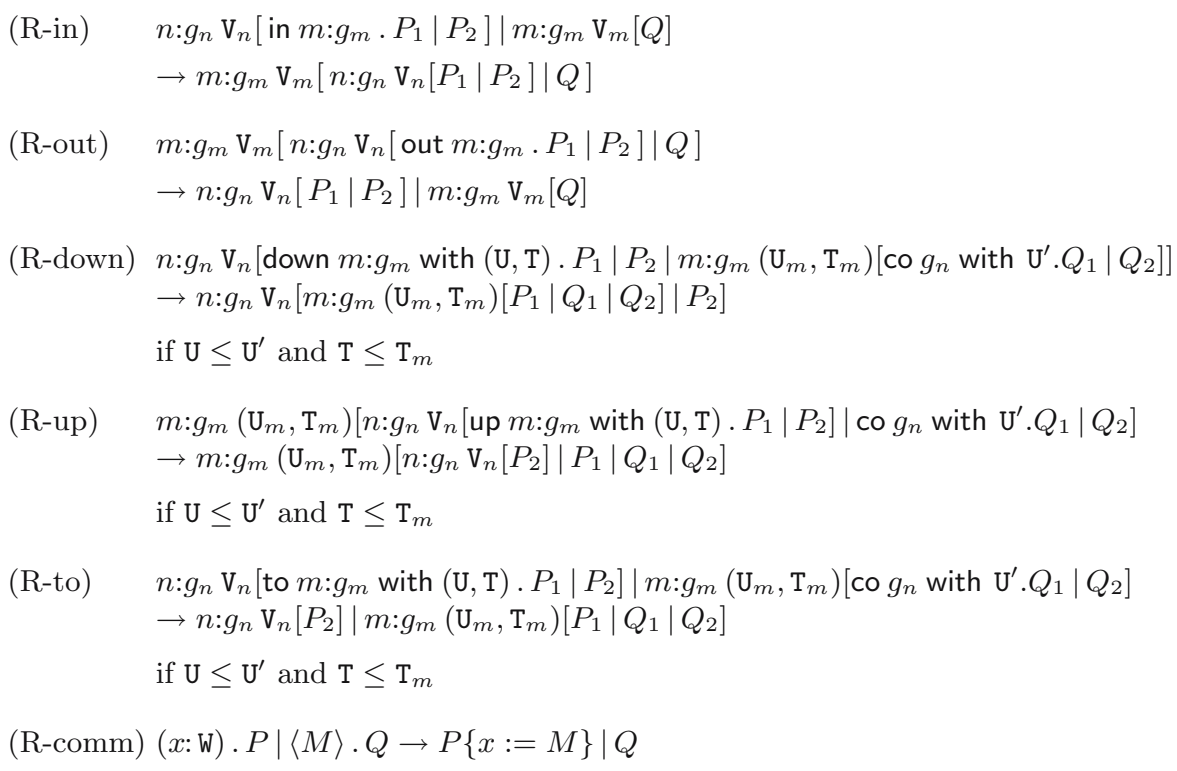

Structural reduction rules:

$$
\begin{array}{ll}
\text { (R-par) } & P \rightarrow Q \Rightarrow P|R \rightarrow Q| R \\
\text { (R-amb) } & P \rightarrow Q \\
\text { (R- } \nu \text {-amb) } & \Rightarrow n: g \mathrm{~V}[P] \rightarrow n: g \mathrm{~V}[Q] \\
\text { (R- } \nu \text {-group) } \quad P^{\prime} \equiv P^{\prime}, \quad P \rightarrow Q, \quad Q \equiv Q^{\prime} & \Rightarrow P^{\prime} \rightarrow Q^{\prime}
\end{array}
$$

Fig. 6. Operational Semantics

Rule (CAP PREFIX) checks that the mobility type of the process matches the type of its prefix, in the same way as rules (INPUT) and (OUTPUT) check that the communication type of the process matches the type of the message. Rules (PAR), (RePl), (Amb Res) and (Grp Res) are standard.

Finally, we present the operational semantics of the calculus. As is common in calculi for distributed computation, the operational semantics relies on a structural congruence and on a reduction relation. The structural congruence equates 
terms whose syntactical differences should be considered inessential; it is defined as the smallest congruence satisfying the laws in Fig. 6. The only non-standard law is $(\nu n) n: g \mathrm{~V}[0] \equiv 0$, which - in the absence of the open capability - is handy for disposing of 'garbage' inactive ambients.

The reduction relation is defined only for closed processes, i.e., processes with no occurrences of free variables; the rules are given in Fig. 6.

The first five rules deal with mobility and can be divided into two groups: those for ambient mobility and those for process mobility. The former are the standard rules of ambient calculi for the in and out capabilities, which can drive ambients into or out of other ambients. The latter are the ones for the down, up and to actions (already found in $\mathbf{M}^{3}$ ), which allow the continuation process to move to a parent, child or sibling ambient, respectively. As discussed earlier, an action of this kind must specify not only the name of the target ambient but also its group, because of the absence of a global mapping from ambients to groups.

In the case of process movements the reduction may take place only if the communication type of the continuation is compatible with the one of the target ambient, and in addition if the ambient contains a suitable co-move letting the process in; both checks make use of the subtyping relations introduced in Definition 1. Note that the co-move is consumed in the reaction and this, in turn, allows the co-move's continuation to synchronize on the entering of a process; moreover, the consumable nature of co-moves allows the implementation of fine-tuned admittance policies.

The last reduction rule is the standard one for communication, local to ambients as in the original ambient calculus.

To get the full reduction relation, the basic reduction rules are then closed under the application of all possible reduction contexts, which are those contexts that are built only by parallel composition, ambient formation, and group and name restriction. Note that no reduction rule can be applied within the continuation of an action prefix, in particular within the body of an input process (before the input itself is performed). Thus, if $C[-]$ is a reduction context and $C[P]$ is a good closed process, no free variable can occur in $P$. Finally, the last rule is standard and states that structurally congruent processes have the same reductions.

\section{Soundness}

The type system satisfies the usual property of subject reduction (Theorem 1), which here, as in all systems of behavioural types, is particularly meaningful. In fact, joined to type safety (Corollary 11), which states the exact correspondence between types and behavioural properties, subject reduction guarantees that any evolution of a system, i.e., any reduction sequence, satisfies the constraints expressed by types. This represents a form of soundness, in the sense that communication and movements of both ambients and processes actually obey the constraints that the type attached to each ambient is supposed to express.

The construction of the proof of the subject reduction theorem starts, as usual, with the statement of generation lemmata, which trivially hold because of the 
evident syntax-directed character of the typing rules. To simplify the statements we only consider for each syntactic category of terms the corresponding type pattern. It is easy to verify that, in all cases, no other type pattern would be possible.

\section{Lemma 1 (Generation Lemma I).}

1. If $\Sigma \vdash \Gamma$ then $\vdash \Sigma$.

2. If $\Sigma \vdash\langle\mathscr{C}, \mathscr{E}\rangle$ then $\Sigma \vdash \gamma$ : group for all $\gamma \in \mathscr{C} \cup \mathscr{E}$.

3. If $\Sigma \vdash \operatorname{cap}(\mathrm{U})$ then $\Sigma \vdash \mathrm{U}$.

4. If $\Sigma \vdash \operatorname{com}(\mathrm{W})$ then $\Sigma \vdash \mathrm{W}$.

5. If $\Sigma \vdash(\mathrm{U}, \mathrm{T})$ then $\Sigma \vdash \mathrm{U}$ and $\Sigma \vdash \mathrm{T}$.

6. If $\vdash \Sigma, x$ : W then $\Sigma \vdash \mathrm{W}$ and $x \notin \operatorname{Dom}(\Sigma)$.

7. If $\Sigma \vdash n:$ amb then $n \in \mathscr{A}$.

8. If $\Sigma \vdash g:$ group then $g \in \mathscr{G}$.

9. If $\Sigma \vdash x: \mathrm{W}$ then $x: \mathrm{W}^{\prime} \in \Sigma$ for some $\mathrm{W}^{\prime} \leq \mathrm{W}$.

10. If $\Sigma \vdash$ in $\alpha: \gamma: \operatorname{cap}(\mathrm{U})$ then $\Sigma \vdash \alpha: \operatorname{amb}$ and $\Sigma \vdash \operatorname{cap}(\mathrm{U})$ and $\gamma \in \mathscr{C}(\mathrm{U})$.

11. If $\Sigma \vdash$ out $\alpha: \gamma: \operatorname{cap}(\mathrm{U})$ then $\Sigma \vdash \alpha:$ amb and $\Sigma \vdash \operatorname{cap}(\mathrm{U})$ and $\gamma \in \mathscr{C}(\mathrm{U})$.

12. If $\Sigma \vdash \operatorname{co} \gamma$ with $\mathrm{U}: \operatorname{cap}\left(\mathrm{U}^{\prime}\right)$ then $\Sigma \vdash \gamma$ : group and $\Sigma \vdash \operatorname{cap}\left(\mathrm{U}^{\prime}\right)$ and $\mathrm{U} \leq \mathrm{U}^{\prime}$.

13. If $\Sigma \vdash \chi \cdot \chi^{\prime}: \operatorname{cap}(\mathrm{U})$ then $\Sigma \vdash \chi: \operatorname{cap}(\mathrm{U})$ and $\Sigma \vdash \chi^{\prime}: \operatorname{cap}(\mathrm{U})$.

\section{Lemma 2 (Generation Lemma II).}

1. If $\Sigma \vdash 0: \mathrm{V}$ then $\Sigma \vdash \mathrm{V}$.

2. If $\Sigma \vdash \chi . P:(\mathrm{U}, \mathrm{T})$ then $\Sigma \vdash \chi: \operatorname{cap}(\mathrm{U})$ and $\Sigma \vdash P:(\mathrm{U}, \mathrm{T})$.

3. If $\Sigma \vdash$ down $\alpha: \gamma$ with V.P : $\mathrm{V}^{\prime}$ then $\Sigma \vdash \alpha$ : amb, $\Sigma \vdash \gamma:$ group and $\Sigma \vdash P: \mathrm{V}$ and $\Sigma \vdash \mathrm{V}^{\prime}$ and $\gamma \in \mathscr{E}\left(\mathrm{V}^{\prime}\right)$.

4. If $\Sigma \vdash$ up $\alpha: \gamma$ with V.P : $\mathrm{V}^{\prime}$ then $\Sigma \vdash \alpha:$ amb, $\Sigma \vdash \gamma:$ group and $\Sigma \vdash P: \mathrm{V}$ and $\Sigma \vdash \mathrm{V}^{\prime}$ and $\gamma \in \mathscr{E}\left(\mathrm{V}^{\prime}\right)$.

5. If $\Sigma \vdash$ to $\alpha: \gamma$ with V.P : $\mathrm{V}^{\prime}$ then $\Sigma \vdash \alpha:$ amb, $\Sigma \vdash \gamma:$ group and $\Sigma \vdash P: \mathrm{V}$ and $\Sigma \vdash \mathrm{V}^{\prime}$ and $\gamma \in \mathscr{E}\left(\mathrm{V}^{\prime}\right)$.

6. If $\Sigma \vdash(x: \mathrm{W}) . P:\left(\mathrm{U}, \operatorname{com}\left(\mathrm{W}^{\prime}\right)\right)$ then $\mathrm{W}=\mathrm{W}^{\prime}$ and $\Sigma, x: \mathrm{W} \vdash P:(\mathrm{U}, \operatorname{com}(\mathrm{W}))$ and $x \notin \Sigma$ and $x \notin(\mathrm{U}, \operatorname{com}(\mathrm{W}))$.

7. If $\Sigma \vdash\langle M\rangle$. P: (U, $\operatorname{com}(\mathrm{W}))$ then $\Sigma \vdash P:(\mathrm{U}, \operatorname{com}(\mathrm{W}))$ and $\Sigma \vdash M: \mathrm{W}$.

8. If $\Sigma \vdash \alpha: \gamma \mathrm{V}[P]: \mathrm{V}^{\prime}$ then $\Sigma \vdash \alpha:$ amb and $\Sigma \vdash \gamma:$ group and $\Sigma \vdash \mathrm{V}^{\prime}$ and $\Sigma \vdash P: \mathrm{V}$.

9. If $\Sigma \vdash P \mid Q: \mathrm{V}$ then $\Sigma \vdash P: \mathrm{V}$ and $\Sigma \vdash Q: \mathrm{V}$.

10. If $\Sigma \vdash ! P: \mathrm{V}$ then $\Sigma \vdash P: \mathrm{V}$.

11. If $\Sigma \vdash(\nu n) P: \mathrm{V}$ then $\Sigma \vdash P: \mathrm{V}$.

12. If $\Sigma \vdash(\nu g) P: \mathrm{V}$ then $\Sigma \vdash P: \mathrm{V}$, and $g \notin \Sigma$ and $g \notin \mathrm{V}$.

Note that the lemmata imply that every valid typing judgement has a unique derivation.

We also need a substitution lemma, which in our case handles the substitution of variables by messages. The application of substitutions to types, messages and processes is standard, while environments need some care. The substitution of 
the variable $x$ by the message $M$ in the environment $\Sigma$ (denoted by $\Sigma\{x:=M\}$ ) is defined by induction on $\Sigma$ :

$$
\begin{aligned}
& \varnothing\{x:=M\} \quad=\varnothing \\
& (\Sigma, y: \mathrm{W})\{x:=M\}= \begin{cases}\Sigma\{x:=M\}, y: \mathrm{W}\{x:=M\} & \text { if } x \neq y \\
\Sigma\{x:=M\} & \text { if } x=y \text { and } M \text { is not a variable } \\
\Sigma\{x:=M\}, M: \mathrm{W}\{x:=M\} & \text { otherwise }\end{cases}
\end{aligned}
$$

In the last case, where $x$ is the same as $y$ and $M$ is a variable, the resulting environment might be non-well-formed (if the variable $M$ occurs in $\Sigma$ ); however, that will never happen with substitutions as used in the paper. Also notice that if $M$ is not a variable, then it is an ambient name, a group name or a capability, but in either case no assignment is allowed for it in environments.

\section{Lemma 3 (Substitution Lemma).}

1. If $\Sigma \vdash \Gamma$ and $x \in \Gamma$ then $x \in \operatorname{Dom}(\Sigma)$.

2. If $\vdash \Sigma, x$ : W and $\Sigma \vdash M: \mathrm{W}$ then $\vdash \Sigma\{x:=M\}$.

3. If $\Sigma, x: \mathrm{W} \vdash \Gamma$ and $\Sigma \vdash M: \mathrm{W}$ then $\Sigma\{x:=M\} \vdash \Gamma\{x:=M\}$.

Proof. The proof of Point (1) by induction on the derivations is standard. Points (2) and (3) can be proved simultaneously by induction on the derivations; we only consider two interesting cases.

The first case is the proof of Point (2) when the last rule applied (in the derivation of the point's first antecedent) is (ENV CONSTR):

$$
\frac{\Sigma, x: \mathrm{W} \vdash \mathrm{W}^{\prime} \quad y \notin \operatorname{Dom}(\Sigma, x: \mathrm{W})}{\vdash \Sigma, x: \mathrm{W}, y: \mathrm{W}^{\prime}}
$$

Then, to the judgement $\Sigma, x: \mathrm{W} \vdash \mathrm{W}^{\prime}$ we may apply the inductive hypothesis of Point (3), with $\Gamma$ instantiated to $\mathrm{W}^{\prime}$, and we obtain $\Sigma\{x:=M\} \vdash \mathrm{W}^{\prime}\{x:=M\}$.

To be able to re-apply the rule (ENv Constr) with $x$ substituted by $M$, and thus to prove the conclusion $\vdash\left(\Sigma, y: \mathrm{W}^{\prime}\right)\{x:=M\}$, we only need to show that the side condition $y \notin \operatorname{Dom}(\Sigma\{x:=M\})$ holds. But this is not hard, since by Lemma 1(11) the well-formedness judgment $\vdash \Sigma, x$ : $\mathrm{W}$ holds, which in turn by Lemma 1(6) implies $x \notin \operatorname{Dom}(\Sigma)$; it follows that $\operatorname{Dom}(\Sigma)=\operatorname{Dom}(\Sigma\{x:=M\})$. From the rule's rightmost premise $y \notin \operatorname{Dom}(\Sigma, x: \mathrm{W})$ in the assumption, we immediately have that $y \notin \operatorname{Dom}(\Sigma)$; thus also $y \notin \operatorname{Dom}(\Sigma\{x:=M\})$.

As a second interesting case we consider the proof of Point (3) when the last rule applied is (INPUT):

$$
\frac{\Sigma, x: \mathrm{W}, y: \mathrm{W}^{\prime} \vdash P:\left(\mathrm{U}, \operatorname{com}\left(\mathrm{W}^{\prime}\right)\right) \quad y \notin(\Sigma, x: \mathrm{W}) \quad y \notin\left(\mathrm{U}, \operatorname{com}\left(\mathrm{W}^{\prime}\right)\right)}{\Sigma, x: \mathrm{W} \vdash\left(y: \mathrm{W}^{\prime}\right) \cdot P:\left(\mathrm{U}, \operatorname{com}\left(\mathrm{W}^{\prime}\right)\right)}
$$

By induction on Point (3), with $\Sigma$ replaced by $\Sigma, y: \mathrm{W}^{\prime}$ and $\Gamma$ instantiated to $P:\left(\mathrm{U}, \operatorname{com}\left(\mathrm{W}^{\prime}\right)\right)$, we obtain $\left(\Sigma, y: \mathrm{W}^{\prime}\right)\{x:=M\} \vdash\left(P:\left(\mathrm{U}, \operatorname{com}\left(\mathrm{W}^{\prime}\right)\right)\right)\{x:=M\}$. As for the two needed side conditions on $y$, observe that by Point (1) $y \notin(\Sigma, x: \mathrm{W})$ 
and $\Sigma \vdash M$ :W imply $y \notin M$; from $y \notin \Sigma$ and $y \notin M$ it follows that $y \notin \Sigma\{x:=$ $M\}$, while the rightmost premise $y \notin\left(\mathrm{U}, \operatorname{com}\left(\mathrm{W}^{\prime}\right)\right)$, again with the condition $y \notin M$, implies that $y \notin\left(\mathrm{U}, \operatorname{com}\left(\mathrm{W}^{\prime}\right)\right)\{x:=M\}$. We may therefore conclude by applying the rule (INPUT), thus obtaining the consequent $\Sigma\{x:=M\} \vdash$ $\left(\left(y: \mathrm{W}^{\prime}\right) \cdot P:\left(\mathrm{U}, \operatorname{com}\left(\mathrm{W}^{\prime}\right)\right)\right)\{x:=M\}$.

The other standard lemmata needed for the proof of subject reduction are those concerning strengthening, weakening, and admissibility of subtyping.

Strengthening is expressed by the first two points of the Lemma below. Point (11) states that if the environment $\Sigma \cup \Sigma^{\prime}$ is well formed and no $x$ occurring in $\Sigma$ 'depends' on $\Sigma^{\prime}$, i.e., is in the domain of $\Sigma^{\prime}$, then $\Sigma$ is separately well formed. Point (2) says that if the judgment $\Sigma \cup \Sigma^{\prime} \vdash \Gamma$ holds, and no $x$ occurring (free) in $\Sigma$ or in $\Gamma$ is in the domain of $\Sigma^{\prime}$, then $\Sigma^{\prime}$ may be disposed of, and the strengthened judgment $\Sigma \vdash \Gamma$ holds.

The Lemma's third point expresses the weakening in the usual form.

\section{Lemma 4 (Strengthening and Weakening Lemma).}

1. If $\vdash \Sigma \cup \Sigma^{\prime}$ and $x \notin \Sigma$ for all $x \in \operatorname{Dom}\left(\Sigma^{\prime}\right)$ then $\vdash \Sigma$.

2. If $\Sigma \cup \Sigma^{\prime} \vdash \Gamma$ and $x \notin \Sigma$ and $x \notin \Gamma$ for all $x \in \operatorname{Dom}\left(\Sigma^{\prime}\right)$ then $\Sigma \vdash \Gamma$.

3. If $\Sigma \vdash \Gamma$ and $\vdash \Sigma^{\prime}$ and $\Sigma \subseteq \Sigma^{\prime}$, then $\Sigma^{\prime} \vdash \Gamma$.

Proof. Points (11) and (2) can be proved simultaneously by induction on derivations. The proof of Point (3) is also by induction on derivations. We only consider a single interesting case, the one in which the last rule applied is (INPUT):

$$
\frac{\Sigma, x: \mathrm{W} \vdash P:(\mathrm{U}, \operatorname{com}(\mathrm{W})) \quad x \notin \Sigma \quad x \notin(\mathrm{U}, \operatorname{com}(\mathrm{W}))}{\Sigma \vdash(x: \mathrm{W}) \cdot P:(\mathrm{U}, \operatorname{com}(\mathrm{W}))}
$$

and, in addition, $x \in \Sigma^{\prime}$.

Let $x^{\prime}$ be a fresh variable; by applying induction to the rule's (leftmost) premise we obtain $\Sigma, x: \mathrm{W}, x^{\prime}: \mathrm{W} \vdash P$ : (U, com(W)), while the rule (ENV) yields $\Sigma, x^{\prime}:$ W $\vdash x^{\prime}$ : W. From these two judgments it follows by Lemma 3(3) (since environments are sets) that the typing $\Sigma, x^{\prime}: \mathrm{W} \vdash P\left\{x:=x^{\prime}\right\}:(\mathrm{U}, \operatorname{com}(\mathrm{W}))$ holds, i.e., is derivable. It is easy to verify that the derivations of $\Sigma, x: \mathrm{W} \vdash P$ : (U, com(W)) and $\Sigma, x^{\prime}: \mathrm{W} \vdash P\left\{x:=x^{\prime}\right\}:(\mathrm{U}, \operatorname{com}(\mathrm{W}))$ are isomorphic; then by the induction hypothesis we obtain $\Sigma^{\prime}, x^{\prime}: \mathrm{W} \vdash P\left\{x:=x^{\prime}\right\}$ : (U, com(W)). By the rule (InPUT) this implies that $\Sigma^{\prime} \vdash\left(x^{\prime}: \mathrm{W}\right) . P\left\{x:=x^{\prime}\right\}:(\mathrm{U}, \operatorname{com}(\mathrm{W}))$, and we are done, since $(x: \mathrm{W}) . P$ and $\left(x^{\prime}: \mathrm{W}\right) . P\left\{x:=x^{\prime}\right\}$ are $\alpha$-equivalent.

\section{Lemma 5 (Admissibility of Subtyping).}

1. If $\Sigma \vdash M: \operatorname{cap}(\mathrm{U})$ and $\mathrm{U} \leq \mathrm{U}^{\prime}$ and $\Sigma \vdash \mathrm{U}^{\prime}$ then $\Sigma \vdash M: \operatorname{cap}\left(\mathrm{U}^{\prime}\right)$.

2. If $\Sigma \vdash P: \mathrm{V}$ and $\mathrm{V} \leq \mathrm{V}^{\prime}$ and $\Sigma \vdash \mathrm{V}^{\prime}$ then $\Sigma \vdash P: \mathrm{V}^{\prime}$.

Proof. Points (11) and (2) are easily proved simultaneously by induction on the derivations. 
We are now finally able to prove subject reduction. The property is expressed in its most natural form, which only holds for closed processes, i.e., processes without free variables.

\section{Theorem 1 (Subject Reduction).}

$$
\text { Let } \vdash P: \mathrm{V} \text {. Then }
$$

1. $P \equiv Q$ implies $\vdash Q: \mathrm{V}$.

2. $P \rightarrow Q$ implies $\vdash Q: \mathrm{V}$.

Proof. The proof is standard, by induction on the derivations of $P \equiv Q$ and $P \rightarrow$ $Q$ using the Weakening, Substitution, Admissibility of Subtyping and Generation Lemmata. We only explicitly present the case of rule (R-up):

$$
\begin{aligned}
& \quad m: g_{m}\left(\mathrm{U}_{m}, \mathrm{~T}_{m}\right)\left[n: g_{n} \mathrm{~V}_{n}\left[\operatorname{up} m: g_{m} \text { with }(\mathrm{U}, \mathrm{T}) . P_{1} \mid P_{2}\right] \mid \operatorname{cog} g_{n} \text { with } \mathrm{U}_{1} \cdot Q_{1} \mid Q_{2}\right] \\
& \quad \rightarrow m: g_{m}\left(\mathrm{U}_{m}, \mathrm{~T}_{m}\right)\left[n: g_{n} \mathrm{~V}_{n}\left[P_{2}\right]\left|P_{1}\right| Q_{1} \mid Q_{2}\right] \\
& \quad \text { if } \mathrm{U} \leq \mathrm{U}_{1} \text { and } \mathrm{T} \leq \mathrm{T}_{m} . \\
& \text { If } \\
& \vdash m: g_{m}\left(\mathrm{U}_{m}, \mathrm{~T}_{m}\right)\left[n: g_{n} \mathrm{~V}_{n}\left[\operatorname{up} m: g_{m} \text { with }(\mathrm{U}, \mathrm{T}) \cdot P_{1} \mid P_{2}\right] \mid \operatorname{co} g_{n} \text { with } \mathrm{U}_{1} \cdot Q_{1} \mid Q_{2}\right]: \mathrm{V}
\end{aligned}
$$

If

then by Lemma 2(8) we get $\vdash \mathrm{V}$ and

$$
\vdash n: g_{n} \mathrm{~V}_{n}\left[\text { up } m: g_{m} \text { with }(\mathrm{U}, \mathrm{T}) . P_{1} \mid P_{2}\right] \mid \operatorname{co} g_{n} \text { with } \mathrm{U}_{1} \cdot Q_{1} \mid Q_{2}:\left(\mathrm{U}_{m}, \mathrm{~T}_{m}\right) \text {. }
$$

By Lemma 2(9) we must have

$$
\begin{array}{r}
\vdash n: g_{n} \mathrm{~V}_{n}\left[\text { up } m: g_{m} \text { with }(\mathrm{U}, \mathrm{T}) . P_{1} \mid P_{2}\right]:\left(\mathrm{U}_{m}, \mathrm{~T}_{m}\right) \\
\vdash \operatorname{cog} g_{n} \text { with } \mathrm{U}_{1} \cdot Q_{1}:\left(\mathrm{U}_{m}, \mathrm{~T}_{m}\right) \\
\vdash Q_{2}:\left(\mathrm{U}_{m}, \mathrm{~T}_{m}\right) .
\end{array}
$$

From (4.2) by Lemma 2(8) we have $\vdash\left(\mathrm{U}_{m}, \mathrm{~T}_{m}\right)$ and

$$
\vdash \text { up } m: g_{m} \text { with }(\mathrm{U}, \mathrm{T}) . P_{1} \mid P_{2}: \mathrm{V}_{n},
$$

which implies by Lemma 29)

$$
\begin{array}{r}
\vdash \text { up } m: g_{m} \text { with }(\mathrm{U}, \mathrm{T}) . P_{1}: \mathrm{V}_{n} \\
\vdash P_{2}: \mathrm{V}_{n} .
\end{array}
$$

Now, by Lemma 2(4) we get $\vdash P_{1}:(\mathrm{U}, \mathrm{T})$ and $\vdash \mathrm{V}_{n}$ and $g_{m} \in \mathscr{E}\left(\mathrm{V}_{n}\right)$. Furthermore, by Lemma 5(2) from $\vdash P_{1}:(\mathrm{U}, \mathrm{T})$ we have $\vdash P_{1}:\left(\mathrm{U}_{m}, \mathrm{~T}_{m}\right)$ since $\mathrm{U} \leq \mathrm{U}_{m}$ and $\mathrm{T} \leq \mathrm{T}_{m}$. From (4.3) by Lemma 2(2) we get $\vdash Q_{1}:\left(\mathrm{U}_{m}, \mathrm{~T}_{m}\right)$.

Rule (АмB) applied to (4.6) gives $\vdash n: g_{n} \mathrm{~V}_{n}\left[P_{2}\right]:\left(\mathrm{U}_{m}, \mathrm{~T}_{m}\right)$ since $\vdash\left(\mathrm{U}_{m}, \mathrm{~T}_{m}\right)$. Rule (PAR) applied to $\vdash n: g_{n} \mathrm{~V}_{n}\left[P_{2}\right]:\left(\mathrm{U}_{m}, \mathrm{~T}_{m}\right), \vdash P_{1}:\left(\mathrm{U}_{m}, \mathrm{~T}_{m}\right), \vdash Q_{1}:\left(\mathrm{U}_{m}, \mathrm{~T}_{m}\right)$, and (4.4) gives

$$
\vdash n: g_{n} \mathrm{~V}_{n}\left[P_{2}\right]\left|P_{1}\right| Q_{1} \mid Q_{2}:\left(\mathrm{U}_{m}, \mathrm{~T}_{m}\right) .
$$

We conclude

$$
\vdash m: g_{m} \mathrm{~V}_{m}\left[n: g_{n} \mathrm{~V}_{n}\left[P_{2}\right]\left|P_{1}\right| Q_{1} \mid Q_{2}\right]: \mathrm{V}
$$

by rule $(\mathrm{AmB})$ since $\vdash \mathrm{V}$. 
Clearly, subject reduction guarantees that in any sequence of reductions every process obeys the constraints imposed by its enclosing ambient. As a matter of fact, a more accurate and stronger property is satisfied: any sub-process of a 'good' process does not only behave in compliance with the policy of its enclosing ambient (if any), but it also complies with the policy against which it has been checked when entering the ambient. Such policy is in general more restrictive than the one of the enclosing ambient, and is expressed by the with component of the co-move that has authorized the movement. Unfortunately this expression completely disappears with its containing co-move as soon as the incoming process is authorized: it thus becomes impossible to formalize the statement that the policy is respected during the whole computation carried out by the process before any further migration.

To overcome this problem, we enrich the process syntax by introducing tagged processes. Tags will be used to record, for each process entering an ambient by consuming a to, down, or up capability, the mobility type U associated with it. To accommodate tags in the syntax of Figure 1 we add the production:

$$
P::=\ldots \quad\left\{\begin{array}{l} 
\\
\{P\}^{\mathrm{U}} \quad \text { tagged process }
\end{array}\right.
$$

Tagging has no influence on process formation and well-typing rules: the type of a tagged process is the one of its untagged version, obtained by dropping all tags occurring in it.

The operational semantics attaches new tags to processes (when needed), but in reduction it considers tagged processes as ordinary ones; i.e., tags are not exploited to enable or disable reduction steps. We always assume that the initial process of a system is untagged and that all tags are generated during reduction. All properties concerning reduction, notably subject reduction, are then unaffected. Nevertheless, the operational semantics must be extended to represent the generation of tags upon process movements and to allow tagged processes to evolve. The basic reduction rules of Fig. 6 are therefore replaced by those given in Fig. 7, where we convene that a process not explicitly tagged may be either untagged or tagged. In the same figure we also give the additional structural congruence rules for dealing with tagged processes. Notice that the tag disappears when tagging 0 or the ambient formation, and that tags commute with all other process constructs except the ones for mobility (of both ambients and processes). For this reason we need to duplicate the movement reduction rules.

The rules are hopefully self-explanatory. We only remark that after a reduction with rule (R-down), (R-up) or (R-to), a tag is attached to the migrating process to record the policy against which it has been checked. Similarly, in rules (R-down $)^{\prime},(\mathrm{R} \text {-up })^{\prime}$ and (R-to)' the tag of the migrating process is changed appropriately.

The structural reduction rules of Fig. 6 are still valid, but we need to extend the notion of reduction contexts to also encompass the occurrence of tagged processes. Thus, we will say that $C[-]$ is a reduction context if its untagged version is a reduction context. 


\section{Structural congruence (additional rules):}

$$
\begin{array}{rlrlrl}
\{0\}^{\mathrm{U}} & \equiv 0 & \left\{\operatorname{cog} \text { with } \mathrm{U}^{\prime} \cdot P\right\}^{\mathrm{U}} & \equiv & \operatorname{cog} \text { with } \mathrm{U}^{\prime} \cdot\{P\}^{\mathrm{U}} \\
\{(x: \mathrm{W}) \cdot P\}^{\mathrm{U}} & \equiv(x: \mathrm{W}) \cdot\{P\}^{\mathrm{U}} & \{\langle M\rangle \cdot P\}^{\mathrm{U}} & \equiv & \langle M\rangle \cdot\{P\}^{\mathrm{U}} \\
\{P \mid Q\}^{\mathrm{U}} & \equiv\{P\}^{\mathrm{U}} \mid\{Q\}^{\mathrm{U}} & \{! P\}^{\mathrm{U}} & \equiv & !\{P\}^{\mathrm{U}} & \\
\{(\nu n) P\}^{\mathrm{U}} & \equiv(\nu n)\{P\}^{\mathrm{U}} & \{(\nu g) P\}^{\mathrm{U}} & \equiv & (\nu g)\{P\}^{\mathrm{U}} \quad(g \notin \mathrm{U}) \\
\left\{n: g \mathrm{~V}[P\}^{\mathrm{U}}\right. & \equiv n: g \mathrm{~V}[P] & P \equiv Q \text { implies } & \{P\}^{\mathrm{U}} \equiv\{Q\}^{\mathrm{U}}
\end{array}
$$

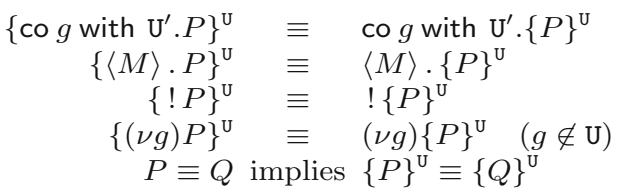

\section{Basic reduction rules:}

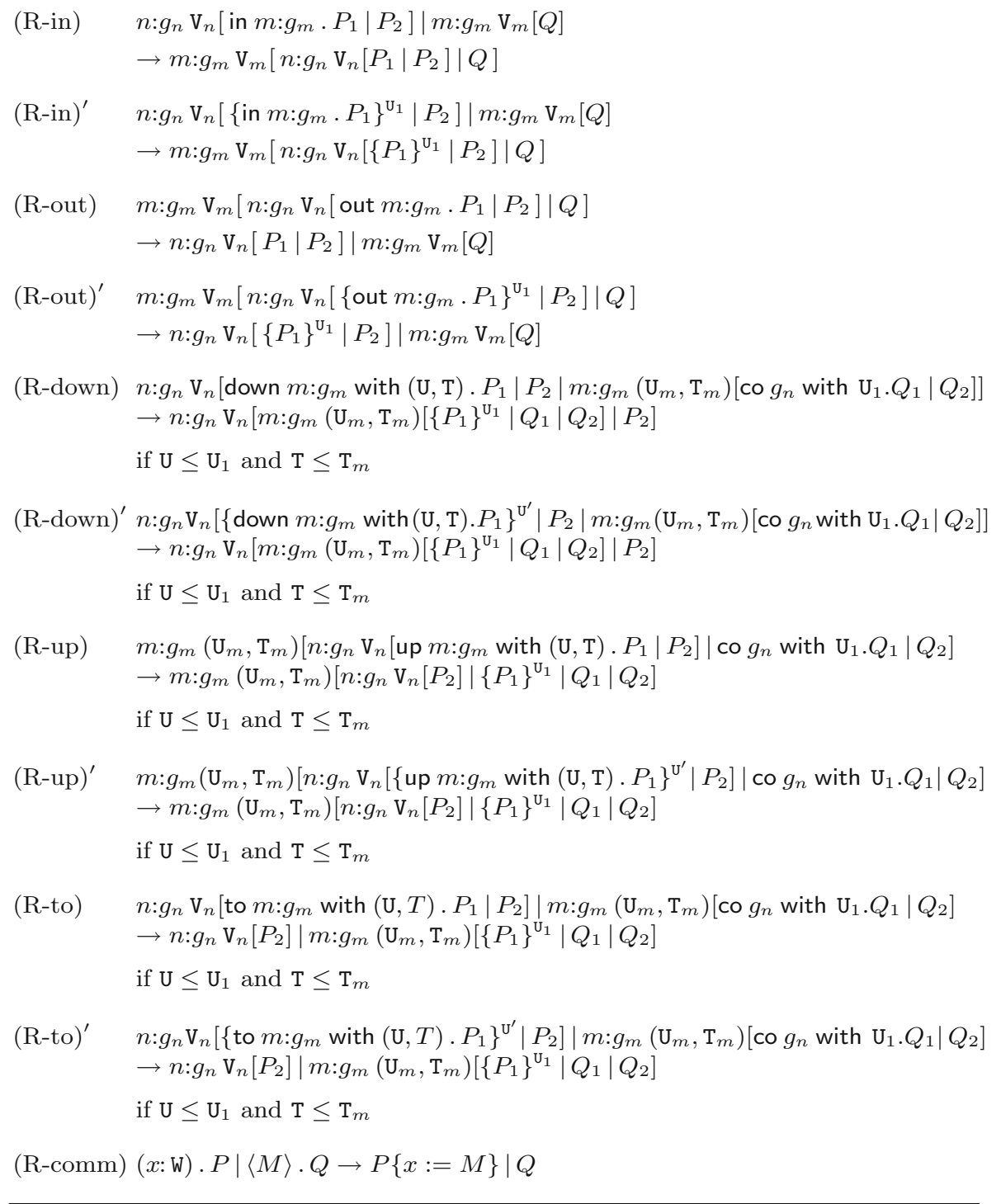

Fig. 7. Operational Semantics with Tags 
The main property of the tag system is that on the one hand the tag assigned to a process during reduction refines the policy of the enclosing ambient, on the other hand it encompasses all the actions the process can perform while running in that ambient. Formally, it is expressed as follows.

Theorem 2 (Correctness of Tagging). If $P$ is a good untagged process and $P \rightarrow{ }^{*} C\left[m: g_{m}\left(\mathrm{U}_{m}, \mathrm{~T}_{m}\right)\left[\left\{P_{1}\right\}^{\mathrm{U}_{1}} \mid P_{2}\right]\right]$, then $\mathrm{U}_{1} \leq \mathrm{U}_{m}$ and $\vdash P_{1}:\left(\mathrm{U}_{1}, \mathrm{~T}_{m}\right)$.

Proof. To show that the thesis holds for all the tags generated by the operational semantics, we prove that it holds as soon as a tag is generated and that the property is preserved by reduction. Formally, we reason by induction on the length of the computation that generates the tag. Since by hypothesis the process $P$ is untagged while the process at the right of the arrow has at least one tag, the reduction sequence must be composed of at least one step (the one that introduces the tag). Let then $Q$ be a process such that

$$
P \rightarrow^{*} Q \rightarrow C\left[m: g_{m}\left(\mathrm{U}_{m}, \mathrm{~T}_{m}\right)\left[\left\{P_{1}\right\}^{\mathrm{U}_{1}} \mid P_{2}\right]\right]
$$

The hypotheses imply that $Q$ is a good process (by Theorem 11) and that all the tags occurring within $Q$ have been generated in the computation from $P$. Therefore, by induction, the thesis holds for all the tags in $Q$.

To prove that the thesis holds also for the tag $\mathrm{U}_{1}$, we reason by induction on the depth of the proof of the last reduction step in (4.7). In the inductive case, the last rule applied to infer the reduction step is a structural reduction rule and therefore the property trivially holds. The base case is when only one of the basic reduction rules of Fig. 7 is applied; we distinguish between process movement and ambient movement or communication.

In the case of a process movement, the tag $\mathrm{U}_{1}$ is generated by the reduction step. We only consider rule (R-down $)^{\prime}$; the other cases can be dealt with similarly. With reference to the notations of Fig. 7, we have $\vdash P_{1}:(\mathrm{U}, \mathrm{T})$ by Lemma 2] (3) and $\mathrm{U}_{1} \leq \mathrm{U}_{m}$ by Lemmata 1(12) and 2(2), because $Q$ is a good process. Also, we have $\mathrm{U} \leq \mathrm{U}_{1}$ and $\mathrm{T} \leq \mathrm{T}_{m}$, otherwise the rule cannot fire. Hence, by Lemma [5](2), we get $\vdash P_{1}:\left(\mathrm{U}_{1}, \mathrm{~T}_{m}\right)$, which proves the thesis.

In the case of a reduction corresponding to an ambient movement or a communication, the tag $\mathrm{U}_{1}$ was already present in $Q$. Again, we only consider one significant case, namely when rule (R-in)' is applied. We refer to the notations of Fig. 7 , but with $n$ and $m$ exchanged. Let $\mathrm{V}_{m}=\left(\mathrm{U}_{m}, \mathrm{~T}_{m}\right)$; then we have $\mathrm{U}_{1} \leq \mathrm{U}_{m}$ and $\vdash$ in $n: g_{n} . P_{1}:\left(\mathrm{U}_{1}, \mathrm{~T}_{m}\right)$ by induction, because the tag $\mathrm{U}_{1}$ was generated previously. Now, $\mathrm{U}_{1} \leq \mathrm{U}_{m}$ still holds because the last reduction step in (4.7) does not change the tag, while $\vdash$ in $n: g_{n} . P_{1}:\left(\mathrm{U}_{1}, \mathrm{~T}_{m}\right)$ implies $\vdash P_{1}:\left(\mathrm{U}_{1}, \mathrm{~T}_{m}\right)$ by Lemma 2(2).

Type safety can now be stated by exploiting tags assigned to processes.

Corollary 1 (Type Safety). Let $Q$ be a good untagged process, and let $Q \rightarrow$ * $C[P] \rightarrow C\left[P^{\prime}\right]$ where $P \rightarrow P^{\prime}$ is obtained by applying one of the basic reduction rules of Fig. 7 . With reference to the notations of Fig. 7 we have: 
1. If $P \rightarrow P^{\prime}$ by (R-in) or (R-out) and $\mathrm{V}_{n}=\left(\mathrm{U}_{n}, \mathrm{~T}_{n}\right)$ then $g_{m} \in \mathscr{C}\left(\mathrm{U}_{n}\right)$.

2. If $P \rightarrow P^{\prime}$ by $(R \text {-in })^{\prime}$ or $(R \text {-out })^{\prime}$ then $g_{m} \in \mathscr{C}\left(\mathrm{U}_{1}\right)$.

3. If $P \rightarrow P^{\prime}$ by (R-up), (R-down) or (R-to) and $\mathrm{V}_{n}=\left(\mathrm{U}_{n}, \mathrm{~T}_{n}\right)$ then $g_{m} \in$ $\mathscr{E}\left(\mathrm{U}_{n}\right)$

4. If $P \rightarrow P^{\prime}$ by $(R \text {-up })^{\prime},(R \text {-down })^{\prime}$ or $(R \text {-to })^{\prime}$ then $g_{m} \in \mathscr{E}\left(\mathrm{U}^{\prime}\right)$.

5. If $P \rightarrow P^{\prime}$ by (R-comm) and $P \equiv(x: \mathrm{W}) \cdot P_{1} \mid\langle M\rangle \cdot P_{2}$ and $C[P] \equiv C^{\prime}\left[n: g_{n}\left(\mathrm{U}_{n}, \mathrm{~T}_{n}\right)\left[(x: \mathrm{W}) . P_{1}\left|\langle M\rangle \cdot P_{2}\right| Q_{1}\right]\right]$ then $\mathrm{T}_{n}=\operatorname{com}(\mathrm{W})$ and $\vdash$ $M: \mathrm{W}$.

Proof. The proofs of cases 1, 3 and 5 follow from the observation that by Theo$\operatorname{rem}[1][P]$ is a good process, therefore by Lemma $2 P$ and all its sub-processes are good processes too. We consider case 5 as an interesting example. In order to type $C[P]$, by Lemma 2(91) we must have $\vdash(x: \mathrm{W}) \cdot P_{1}:\left(\mathrm{U}_{n}, \mathrm{~T}_{n}\right)$ and $\vdash\langle M\rangle \cdot P_{2}:\left(\mathrm{U}_{n}, \mathrm{~T}_{n}\right)$ which, by Lemma 2(7) and (8), imply that $\operatorname{com}(\mathrm{W})=\mathrm{T}_{n}$ and $\vdash M$ : W.

For cases 2 and 4 notice that by Theorem 2 if $\left\{P_{1}\right\}^{\mathrm{U}_{1}}$ is a sub-process of $P$ then $\vdash P_{1}:\left(\mathrm{U}_{1}, \mathrm{~T}\right)$ for some $\mathrm{T}$.

We can therefore conclude that ambient and process moves always respect the mobility rights represented by types, and that the types of messages exchanged in communications always match.

As an example, we apply the previous results to the scenario described in Section 2 and prove some behavioural and security properties of the journals.

Proposition 1. The ambient $j r n_{k}$ is immobile and can send processes only to ambients of groups $\left\{g_{t d l_{1}}, \ldots, g_{t d l_{m}}\right\}$. Also, whenever pub is the only ambient of group $g_{p u b}$, a process entering $j r n_{k}$ from an ambient of group $g_{t d l_{i}}$ is allowed to send a process to an ambient of the same group, while a process from an ambient of group $g_{d l_{i}}$ cannot send processes.

Proof. The ambient $j r n_{k}$ is immobile since its internal process is well typed with type $\left(\left\langle\varnothing,\left\{g_{t d l_{1}}, \ldots, g_{t d l_{m}}\right\}\right\rangle, \operatorname{com}(\right.$ paper $\left.)\right)$. Therefore by Theorem 1 and Corollary 1(11) and (2) no action driving it into or out of another ambient is possible. Similarly, by Theorem 1 and Corollary 1(3) and (4), each process inside $j r n_{k}$ can send processes only to ambients belonging to the set of groups $\left\{g_{t d l_{1}}, \ldots, g_{t d l_{m}}\right\}$.

Notice that the initial co-moves in the ambient $j r n_{k}$ only let in processes coming from ambients of group $g_{p u b}$, while the co-moves in the ambient $p u b$ only let in 'idle' processes, i.e., processes that can be tagged by $\langle\varnothing \varnothing, \varnothing\rangle$. So, the ambient $j r n_{k}$ can receive from the ambient pub only two kinds of co-moves (see process MGR): ! co $g_{t d l_{i}}$ with $\left\langle\varnothing \varnothing,\left\{g_{t d l_{i}}\right\}\right\rangle$ and ! $\operatorname{cog}_{d l_{i}}$ with $\langle\varnothing, \varnothing\rangle$. Therefore, a process $P$ entering $j r n_{k}$ from an ambient of group $g_{t d l_{i}}$ will be tagged by $\left\langle\varnothing,\left\{g_{t d l_{i}}\right\}\right\rangle$ and so by Theorem 2 we get $\vdash P$ : ( $\left\langle\varnothing,\left\{g_{t d l_{i}}\right\}\right\rangle$, com(paper)): this, by Theorem 1 and Corollary 1(3) and (4), means that $P$ can send processes to ambients of group $g_{t d l_{i}}$. On the other hand, a process entering $j r n_{k}$ from an ambient of group $g_{d l_{i}}$ will be tagged by $\langle\varnothing \varnothing, \varnothing\rangle$ and so it cannot send processes. 


\section{An Exemplifying Application: The Train Scenario}

In this section we focus on the modelling of a public transportation system, the train introduced by [9], as a nice illustration of the issues related to the control of mobility.

We want to represent a railway network connecting a set of different places (e.g., cities) in the world. Trains move between stations, travellers may get into and off trains only at stations and cannot drive them (no hijacking is possible). The number of passengers in a train at any given instant cannot exceed the number of seats; a passenger takes a seat on boarding and releases it on getting off. Each train has a fixed route.

For the sake of simplicity, we assume that:

- There is a top-level untrusted ambient world, which includes stations, travellers, and some other unspecified process $S$ (e.g. other means of transport); it has mobcom type $\mathrm{V}_{w}$, but no assumption can be made on it.

- In our intended representation different stations should be found within different cities or localities, and moving from one city to another would only be possible by train. The presence of cities would however increase the size of the example in a trivial manner, without providing more insights; we therefore place stations directly within world, although in this way travellers appear to use a train to end up in the same ambient world which they started from.

- There are only two stations $s t A$ and $s t B$, and one train TRAIN commuting between them. Initially, the train is within st $A$.

Stations and trains are represented by ambient processes; travellers are represented by simple processes; the number of free seats in a train is represented by the multiplicity of the co-actions allowing to get into the train.

Since there is no communication in any considered ambient except at most in the world, we will write for all other ambients mobcom types $\langle\mathscr{C}, \mathscr{E}\rangle$ instead of $(\| \mathscr{C}, \mathscr{E}\rangle$, shh $)$.

Stations are immobile ambients of group $g_{s t}$, and can have travellers both going down into the trains (of group $g_{t r}$ ) or up into the world (of group $g_{w}$ ); they can be crossed by trains, and can receive travellers with different rights both from train and from the outside world. Stations contain also an ambient checkOut instrumental to preserving the condition of having at most $\mathrm{n}$ passengers on the train. Each station thus always contains the process:

$$
\mathrm{STATP} \triangleq ! \operatorname{co} g_{w} \text { with } \mathrm{U}_{\text {from }} \mid ! \operatorname{co} g_{t r} \text { with } \mathrm{U}_{t o} \mid \text { checkOut: } g_{\text {check }} \mathrm{U}_{\text {from }}\left[! \operatorname{co} g_{s t} \text { with } \mathrm{U}_{\text {from }}\right]
$$

where $\mathrm{U}_{\text {from }}=\left\langle\varnothing,\left\{g_{\text {tr }}\right\}\right\rangle, \mathrm{U}_{t o}=\left\langle\varnothing \varnothing,\left\{g_{w}, g_{\text {check }}\right\}\right\rangle$. Therefore the process STATP can be typed by the mobility type $\mathrm{U}_{s t}=\left\langle\varnothing \varnothing,\left\{g_{t r}, g_{w}, g_{\text {check }}\right\}\right\rangle$.

The mobility types $\mathrm{U}_{\text {from }}$ and $\mathrm{U}_{t o}$ specify the behaviours of a passenger respectively in the departure station, when going to board a train, and in the arrival station, when going to exit the station into the outside world or city and to send a notification (through the ambient checkOut) that a seat becomes free. 
As an immediate application of Theorem 2 and Lemmata 11 and 2 passenger behaviours must respect this policy, so for example a passenger entering the station from the world cannot send a notification to the ambient checkOut.

The train is an ambient which can cross stations, send traveller processes into stations and receive at most $\mathrm{n}$ passengers from stations, provided they behave as good passengers (and not, for example, as drivers). The train can also receive the notification of free seats from the ambient checkOut.

$$
\begin{aligned}
\text { TRAIN } \triangleq & \operatorname{tr}: g_{t r}\left(\left\langle\left\{g_{s t}\right\},\left\{g_{s t}\right\}\right\rangle\right)[\underbrace{\operatorname{co} g_{s t} \text { with } \mathrm{U}_{p s n g}|\ldots| \operatorname{co} g_{s t} \text { with } \mathrm{U}_{p s n g} \mid}_{\mathrm{n}} \\
& \left.! \operatorname{co} g_{\text {check }} \text { with } \mathrm{U}_{p s n g} \mid \text { ! out } s t A: g_{s t} \text {. in } s t B: g_{s t} \text {. out } s t B: g_{s t} \text {. in stA: } g_{s t}\right]
\end{aligned}
$$

where $\mathrm{U}_{p s n g}=\left\langle\varnothing,\left\{g_{s t}\right\}\right\rangle$.

A traveller is represented by a parametric process TRAVELLER $(s r c, d s t)$ which from some unspecified place in the world enters the station src to become a passenger of a train that takes him to the station $d s t$ :

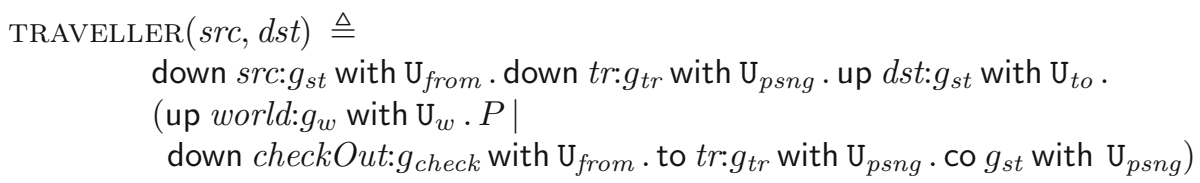

where $\mathrm{U}_{w}$ are the rights of passengers in the world. The traveller after exiting the train sends a co-move to the train using the ambient checkOut.

The initial configuration is:

$$
\begin{gathered}
(\nu s t A, s t B)\left(\text { world: } g _ { w } ( \mathrm { V } _ { w } ) \left[! \operatorname{co} g_{s t} \text { with } \mathrm{U}_{w}|S| \operatorname{TRVLRS}(s t A, s t B)\right.\right. \\
\qquad \begin{array}{c}
\left|s t A: g_{s t}\left(\mathrm{U}_{s t}\right)[\operatorname{TRAIN} \mid \mathrm{STATP}]\right| \operatorname{st} B: g_{s t}\left(\mathrm{U}_{s t}\right)[\mathrm{STATP}] \\
\mid \operatorname{TRVLRS}(s t B, s t A)])
\end{array}
\end{gathered}
$$

where $S$ is unknown (the world can be dangerous!) and TRVLRS(src, $d s t$ ) is a parallel composition of TRAVELLER $(s r c, d s t)$ processes. The world accepts passengers from stations, since it contains ! co $g_{s t}$ with $\mathrm{U}_{w}$.

A bad passenger willing to get off the train when this is not in a station, though it may be statically well-typed, is dynamically not allowed to do so. Suppose the bad passenger is represented by the process

$$
\text { BADPSNG } \triangleq \text { down } t r: g_{t r} \text { with } \mathrm{U}_{b a d} \text {. up world: } g_{w} \text { with } \mathrm{U}_{w} \text {. BP }
$$

By assuming $\Sigma \vdash \mathrm{BP}: \mathrm{U}_{w}$ one may derive the typing

$$
\Sigma \vdash \text { up world: } g_{w} \text { with } \mathrm{U}_{w} \text {. BP : } \mathrm{U}_{b a d} \quad \text { with } \mathrm{U}_{b a d} \triangleq\left\langle\left\langle\varnothing,\left\{g_{w}\right\}\right\rangle\right.
$$

Observe that the mobility type $\mathrm{U}_{b a d}$ characterizes a process that, once boarded the train, wants to go from it directly into the world. From the above, we may infer the typing $\Sigma \vdash$ BADPSNG : $\mathrm{U}_{s t}$, since for that it is enough that $\mathrm{U}_{s t}$ allows the process to get into the train, i.e., $g_{t r} \in \mathscr{E}\left(\mathrm{U}_{s t}\right)$.

The process BADPSNG is therefore statically allowed to stay within a station, as for example in the well-typed term $s t A: g_{s t} \mathrm{U}_{s t}[$ BADPSNG $\mid$ TRAIN]. Nevertheless, 
when trying at runtime to get into the train, the process is blocked. As a matter of fact, for the action down tr: $g_{t r}$ with $\mathrm{U}_{\text {bad }}$ to fire, it is required that $\mathrm{U}_{b a d} \leq \mathrm{U}_{\text {psng }}$, which is not the case since $\mathrm{U}_{b a d}=\left\langle\varnothing,\left\{g_{w}\right\}\right\rangle$ while $\mathrm{U}_{p s n g}=\left\langle\left\langle\varnothing,\left\{g_{s t}\right\}\right\rangle\right)$ : the type $\mathrm{U}_{\text {bad }}$ allows going into the world while $\mathrm{U}_{\text {psng }}$ does not.

This should have been somehow expected, because in our calculus the dynamic checks, performed when co-moves are consumed, are assigned the very task of controlling that mobile processes either respect some given policies expressed through types, or are blocked. Notice that all the previous properties are guaranteed by exploiting in the operational semantics only information local to the involved processes.

A similar scenario has already been modelled in [9|19|14]. In the first two cases, the mobility control is implemented by informing the passenger when the train has reached the station at which he wants to get off. More specifically, in [9 a new primitive for ambient renaming is exploited. Intuitively, the train ambient takes a suitable name to implicitly inform the passengers when it has arrived at a certain station and to allow them to get in or off, while it takes a name unknown to passengers when it is moving (in this way passengers cannot get in or off the train). In [19], mobility policies are implemented through guardians, i.e., components attached to ambients for monitoring inner activities and interaction with the external environment. When the train arrives at a station, the attached guardian allows passengers to get in; in addition, the train generates a suitable ambient called announcement that informs the passengers of the arrival at a certain station and guides the passengers willing to get off. In [14, the mobility control is performed by exploiting dynamic checks to ensure that mobile processes willing to get in an ambient do respect some fixed policies expressed through types.

\section{Type Inference}

An inference algorithm for a typed calculus takes a raw term, i.e. a simplified form of term with no or only partial type annotations, and reconstructs an ordinary typed term along with a valid typing judgment for it. The first decision to be taken when designing an algorithm therefore concerns the syntax for raw terms.

In our calculus the naive approach of erasing all type annotations does not work, because in this way a typing cannot always be sensibly reconstructed; we have to leave in the term some type information, which - by taking part in the reduction rules - actually 'implements' some specified behaviour of the modelled system.

In particular, we have chosen to erase the type in the input binder and to eliminate as many mobcom annotations as reasonable (those in the with component of the prefixes up, down and to, and in the ambient construct); on the other hand, the group assigned to an ambient occurrence and the group and the mobility type within a co-move are kept, because these annotations define the policies and the mobility constraints established by the designer of the applica- 


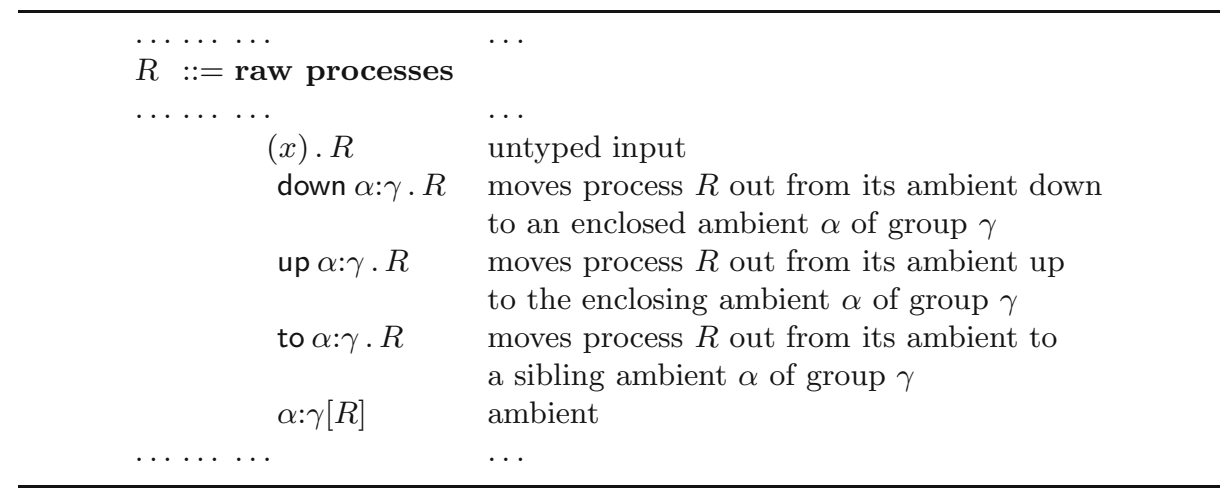

Fig. 8. Raw Processes

tion. The formal definition of raw terms is given in Fig. 8, where the missing parts are as in Fig. 11. We omit the obvious definition of the untyped version $|P|$ of a typed term $P$.

An analysis of the typing rules shows that they do not directly provide an algorithm for inferring the typing of a term, because of three distinct problems. The first is the implicit weakening present in the rule (ENV), coupled with the fact that the different premises of a single rule must share the same environment. This is well known: in such cases, a more algorithmic system can be obtained by delaying the application of weakening until the end of the inference process and by admitting different environments in different premises.

The second problem arises from the fact that there is no uniqueness of typing: in the rule (NULL), for instance, the type of 0 has no relationship with the term. The standard approach consists in the introduction of type variables; this alone, however, is not sufficient here, since in some rules the type variables occurring in the conclusion are limited in their range by conditions in the premises. For instance, in the rule (IN-I) (corresponding to rule (IN)) the variable $u$ of the capability type scheme $\operatorname{cap}(u)$ that types the conclusion must satisfy the constraint of being greater than or equal to the mobility type $\langle\{\{\gamma\}, \varnothing\rangle$. We cope with this difficulty by employing, jointly with type variables, the technique of delaying the solution by simply recording the constraints: these will then be solved at the very end (see e.g. Chapter 22 of 34).

The third problem comes from having dependent types, i.e., possible occurrences of group variables and group names within type expressions: when a variable or name $\gamma$ is being bound, as in rules (INPUT) and (GRP RES), one must check that such $\gamma$ does not occur in the current environment and type. In the type inference this condition is expressed by adding non-occurrence constraints, i.e., constraints of the form $\gamma \notin \mathcal{I}$ where $\mathcal{I}$ is a set of group variables or group names. A substitution satisfies the constraint $\gamma \notin \mathcal{I}$ when, applied to all the variables in $\mathcal{I}$, returns types without occurrences of $\gamma$.

Fig. 9 defines type schemes, i.e. a type syntax augmented with type variables (denoted by lowercase letters): more specifically, we introduce three distinct sets 


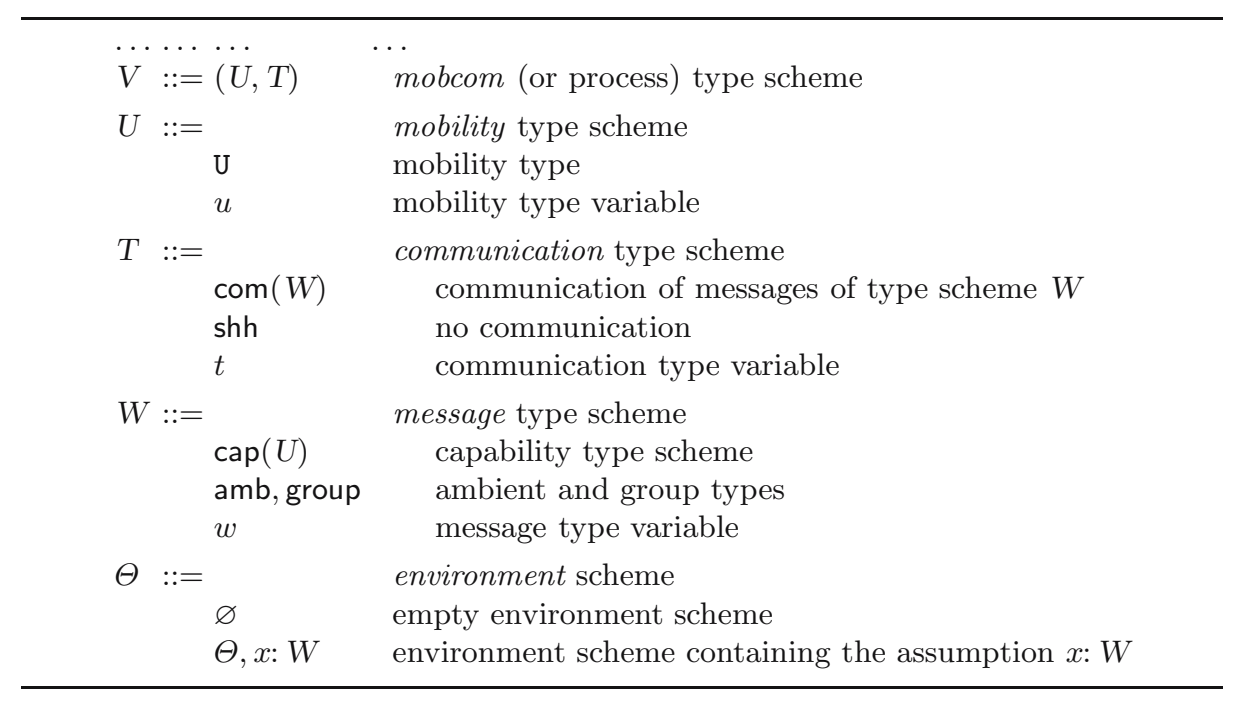

Fig. 9. Type Schemes

of variables respectively for mobility types, message types and communication types. We use $\xi$ to range over communication, message and mobility type variables and $\Xi$ to range over communication, message and mobility type schemes.

We remark that types in raw terms can belong only to the original type syntax, while the inference algorithm can infer (and annotate terms with) type schemes. More precisely, the inference algorithm builds process schemes, environment schemes and sets of constraints, defined respectively as follows.

Definition 2. 1. A process scheme is defined by the same syntax as the one of a process in Fig. 1, except that all type decorations are type schemes instead of types.

2. An environment scheme is defined by the same syntax as the one of an environment in Fig. 2, except that all predicates are type schemes instead of types.

3. A set of constraints is a set whose elements may be equalities and inequalities (w.r.t. the relation $\leq$ ) between type schemes, and non-occurrence constraints.

We use $S$ to range over process schemes and $\mathcal{C}$ to range over sets of constraints.

The input of the algorithm is either a message $M$ or a raw term $R$. In the first case the output consists of: an environment scheme $\Theta$, the message $M$, a message type scheme $W$ and a set of constraints $\mathcal{C}$. In the second case the output is given by: an environment scheme $\Theta$, a process scheme $S$, a mobcom type scheme $V$ and a set of constraints $\mathcal{C}$. We respectively use the notations:

$$
M \Longrightarrow\left\langle\Theta \vdash_{I} M: W \| \mathcal{C}\right\rangle \quad \text { and } \quad R \Longrightarrow\left\langle\Theta \vdash_{I} S: V \| \mathcal{C}\right\rangle
$$

It is easy to verify, by looking at the inference rules in Fig. 10, 11 and 12, that the elements of a set of constraints are of the form $\Xi=\Xi^{\prime}$ or $U \leq u$ or $\gamma \notin \mathcal{I}$. 
To relate type inference with type assignment, type variables must be replaced by types of the respective kinds. More precisely, a solution of a set of constraints is defined as follows.

Definition 3. 1. A ground substitution is a total mapping from mobility variables to mobility types, from message variables to message types and from communication variables to communication types.

2. A solution of a set of constraints $\mathcal{C}$ is a ground substitution $\varsigma$ such that

- if $\Xi=\Xi^{\prime} \in \mathcal{C}$ then $\varsigma(\Xi)=\varsigma\left(\Xi^{\prime}\right)$

- if $U \leq u \in \mathcal{C}$ then $\varsigma(U) \leq \varsigma(u)$;

- if $\gamma \notin \mathcal{I} \in \mathcal{C}$ then $\gamma \notin \varsigma(\xi)$ for all $\xi \in \mathcal{I}$.

As usual, the application of $\varsigma$ to $\Upsilon$, written $\varsigma(\Upsilon)$, with $\Upsilon \in\{\xi, \Xi, U, \ldots\}$, denotes the term obtained by replacing all the variables $\xi$ occurring in $\Upsilon$ with $\varsigma(\xi)$.

The handling of constraints will be explained later; suffice it now to say that we will present an algorithm solve which accepts as input a set of constraints $\mathcal{C}$ and either fails or outputs a solution, as stated in Proposition 4.

For the description of the inference rules some preliminary definitions are necessary. We need to eliminate from sets of typing assumptions those whose subjects are not variables: let then $\Omega$ be a set of typing assumptions whose subjects are names or variables: $\Omega \Downarrow$ is defined as the maximum subset containing only assumptions whose subjects are variables, i.e., $\Omega \Downarrow=\{x: W \mid x: W \in \Omega\}$.

In defining the combination of two environment schemes our choice is that whenever they respectively contain two (generally different) assumptions with the same subject, we take the assumption found in the leftmost environment and add to the set of constraints the equality between the two predicates. More precisely we define:

- the combination $\Theta \triangleright \Theta^{\prime}$ of two environment schemes $\Theta$ and $\Theta^{\prime}$ :

$$
\Theta \triangleright \Theta^{\prime}=\{x: W \mid x: W \in \Theta\} \cup\left\{x: W \mid x \notin \operatorname{Dom}(\Theta) \& x: W \in \Theta^{\prime}\right\}
$$

- the set of constraints $\Theta \diamond \Theta^{\prime}$ generated by the combination of two environment schemes $\Theta$ and $\Theta^{\prime}$ :

$$
\Theta \diamond \Theta^{\prime}=\left\{W=W^{\prime} \mid x: W \in \Theta \& x: W^{\prime} \in \Theta^{\prime}\right\} .
$$

Clearly, combination might be defined with the roles of $\Theta$ and $\Theta^{\prime}$ exchanged; in any case the constraints obtained would be the same, as is obvious from the symmetric nature of the operator $\diamond$. It is also easy to verify that every solution of the set of constraints, when applied to the combination of the two originating environments, is the same as the union of its application to the environments themselves, as stated by the following proposition.

Proposition 2. If $\varsigma$ is a solution of $\Theta \diamond \Theta^{\prime}$ then $\varsigma\left(\Theta \triangleright \Theta^{\prime}\right)=\varsigma(\Theta) \cup \varsigma\left(\Theta^{\prime}\right)$.

The inference rules are given in Fig. 10, 11 and 12. Type variables that do not occur in the premises are assumed to be fresh; in this way, when different 


$$
\begin{aligned}
& \frac{n \in \mathscr{A}}{n \Longrightarrow\left\langle\varnothing \vdash_{I} n: \mathrm{amb} \| \varnothing\right\rangle}\left(\text { AmB Const-I) } \frac{g \in \mathscr{G}}{g \Longrightarrow\left\langle\varnothing \vdash_{I} g: \text { group } \| \varnothing\right\rangle}\right. \text { (GRP Const-I) } \\
& \overline{x \Longrightarrow\left\langle\{x: w\} \vdash_{I} x: w^{\prime} \| w \leq w^{\prime}\right\rangle} \quad(\text { ENV-I }) \\
& \overline{\text { in } \alpha: \gamma \Longrightarrow\left\langle\{\alpha: \text { amb, } \gamma: \text { group }\} \Downarrow \vdash_{I} \text { in } \alpha: \gamma: \operatorname{cap}(u) \|\{\langle\{\gamma\}, \varnothing\rangle \leq u\}\right\rangle} \quad(\text { IN-I) } \\
& \overline{\text { out } \alpha: \gamma \Longrightarrow\left\langle\{\alpha: \text { amb, } \gamma: \text { group }\} \Downarrow \vdash_{I} \text { out } \alpha: \gamma: \operatorname{cap}(u) \|\{\langle\{\{\gamma\}, \varnothing\rangle \leq u\}\rangle\right.} \quad \text { (OuT-I) } \\
& \overline{\operatorname{co} \gamma \text { with } \mathrm{U} \Longrightarrow\left\langle\{\gamma \text { : group }\} \Downarrow \cup\{x \text { : group } \mid x \in \mathrm{U}\} \vdash_{I} \operatorname{co} \gamma \text { withU }: \operatorname{cap}(u) \|\{\mathrm{U} \leq u\}\right\rangle} \text { (CO-I) } \\
& \frac{\chi \Longrightarrow\left\langle\Theta \vdash_{I} \chi: W \| \mathcal{C}\right\rangle \quad \chi^{\prime} \Longrightarrow\left\langle\Theta^{\prime} \vdash_{I} \chi^{\prime}: W^{\prime} \| \mathcal{C}^{\prime}\right\rangle}{\chi \cdot \chi^{\prime} \Longrightarrow\left\langle\Theta \triangleright \Theta^{\prime} \vdash_{I} \chi \cdot \chi^{\prime}: \operatorname{cap}(u) \| \mathcal{C} \cup \mathcal{C}^{\prime} \cup\left\{W=\operatorname{cap}(u), W^{\prime}=\operatorname{cap}(u)\right\} \cup \Theta>\Theta^{\prime}\right\rangle} \text { (РAтH-I) }
\end{aligned}
$$

Fig. 10. Type Inference Rules for Messages

$$
\begin{aligned}
& \overline{0 \Longrightarrow\left\langle\varnothing \vdash_{I} 0:(u, t) \| \varnothing\right\rangle} \quad(\text { NULL-I }) \\
& \frac{\chi \Longrightarrow\left\langle\Theta \vdash_{I} \chi: W \| \mathcal{C}\right\rangle \quad R \Longrightarrow\left\langle\Theta^{\prime} \vdash_{I} S:(u, t) \| \mathcal{C}^{\prime}\right\rangle}{\chi \cdot R \Longrightarrow\left\langle\Theta \triangleright \Theta^{\prime} \vdash_{I} \chi \cdot S:(u, t) \| \mathcal{C} \cup \mathcal{C}^{\prime} \cup\{W=\operatorname{cap}(u)\} \cup \Theta \triangleright \Theta^{\prime}\right\rangle} \quad \text { (CAP PREFIX-I) } \\
& R \Longrightarrow\left\langle\Theta \vdash_{I} S: V \| \mathcal{C}\right\rangle \\
& \text { down } \alpha: \gamma \cdot R \Longrightarrow\left\langle\{\alpha \text { : amb, } \gamma: \text { group }\} \Downarrow \triangleright \Theta \vdash_{I} \text { down } \alpha: \gamma \text { with } V . S:(u, t) \| \mathcal{C}^{\prime}\right\rangle \\
& \text { where } \mathcal{C}^{\prime}=\mathcal{C} \cup\{\langle\varnothing,\{\gamma\}\rangle \leq u\} \cup\{\alpha \text { : amb, } \gamma \text { : group }\} \Downarrow \diamond \Theta \\
& R \Longrightarrow\left\langle\Theta \vdash_{I} S: V \| \mathcal{C}\right\rangle \\
& \overline{\text { up } \alpha: \gamma . R \Longrightarrow\left\langle\{\alpha \text { : amb, } \gamma: \text { group }\} \Downarrow \triangleright \Theta \vdash_{I} \text { up } \alpha: \gamma \text { with } V . S:(u, t) \| \mathcal{C}^{\prime}\right\rangle} \\
& \text { where } \mathcal{C}^{\prime}=\mathcal{C} \cup\{\langle\varnothing,\{\gamma\}\rangle \leq u\} \cup\{\alpha \text { : amb, } \gamma \text { : group }\} \Downarrow \diamond \Theta \\
& R \Longrightarrow\left\langle\Theta \vdash_{I} S: V \| \mathcal{C}\right\rangle \\
& \text { to } \alpha: \gamma . R \Longrightarrow\left\langle\{\alpha: \text { amb, } \gamma: \text { group }\} \Downarrow \triangleright \Theta \vdash_{I} \text { to } \alpha: \gamma \text { with } V . S:(u, t) \| \mathcal{C}^{\prime}\right\rangle \\
& \text { where } \mathcal{C}^{\prime}=\mathcal{C} \cup\{\langle\varnothing,\{\gamma\}\rangle \leq u\} \cup\{\alpha \text { : amb, } \gamma \text { : group }\} \Downarrow \diamond \Theta
\end{aligned}
$$

Fig. 11. Type Inference Rules for Raw Processes I

derivations are pasted together in a multiple-premise rule, their variables are all distinct.

Rules (Amb Const-I) and (GRP Const-I) are very similar to (AMB Const) and (GRP CONST): the only difference is in the inferred environment, which is empty as is the constraint set. Similarly in (ENV-I) the minimal environment is 


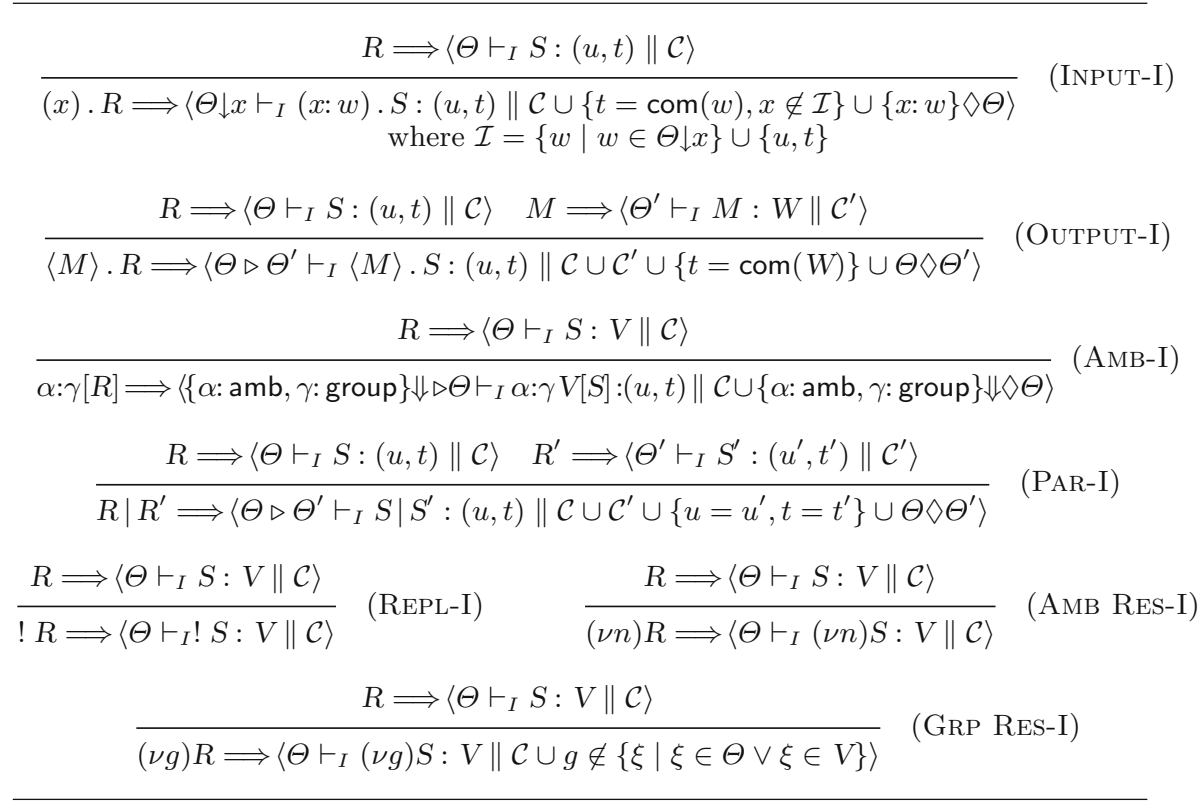

Fig. 12. Type Inference Rules for Raw Processes II

inferred; the inferred type scheme is a fresh message type variable which must be greater than or equal to the message type variable assumed for the term variable in the environment.

Rules (IN-I) and (OUT-I) are the first interesting cases, because they show how the typing rules are adapted to the inference framework. The condition $\gamma \in$ $\mathscr{C}$ in rules (IN) and (OUT) becomes the constraint $\langle\{\gamma\}, \varnothing\rangle \leq u$, where $u$ is the fresh mobility type variable such that $\operatorname{cap}(u)$ is the type scheme inferred for the capability. The assumptions $\alpha$ : amb and $\gamma$ : group are added to the environment scheme only if $\alpha, \gamma$ are variables. To avoid writing several different rules we build the set $\{\alpha$ :amb, $\gamma$ : group $\}$ and then obtain an environment scheme by filtering it through the operator $\Downarrow$.

In rule (Co-I) we add to the environment (scheme) all the variables that occur in the mobility type $U$ and we assume for them the type group. The resulting mobility type scheme is $\operatorname{cap}(u)$, where $u$ is a fresh variable constrained to be greater than or equal to $\mathrm{U}$.

In rule (PATH-I) it is easy to verify that $W$ and $W^{\prime}$ can be either message type variables or capability type schemes containing only mobility variables. Besides, $W$ (respectively $W^{\prime}$ ) is a message type variable iff $\chi$ (respectively $\chi^{\prime}$ ) is a variable. In both cases it is necessary that the mobility rights of the path are equal to those of the components. This is accomplished by requiring that $W=W^{\prime}=\operatorname{cap}(u)$, where the fresh variable $u$ represents the mobility rights of $\chi, \chi^{\prime}$ and $\chi \cdot \chi^{\prime}$. We keep track of the previous constraints $\mathcal{C}$ and $\mathcal{C}^{\prime}$ by adding them to the new constraint set. In addition, the environment schemes of the 
premises are combined using the operator $\triangleright$ and the equalities generated by the application of $\diamond$ to these environments are added to the set of constraints.

For the 0 process we derive the parametric mobcom type scheme $(u, t)$ from the empty environment and we do not require any constraint.

Rule (CAP PrefiX-I) is similar to rule (PATH-I): the message type scheme variable representing the mobility rights of the capability must be equal to $\operatorname{cap}(u)$, where the mobility variable $u$ represents the mobility rights of the whole process.

In the rules for the process-moving prefixes (Down-I)-(TO-I) we decorate the prefix (after the keyword with) with the mobcom type scheme of the process to be sent. For the type scheme of the whole process we only require that it expresses the right to send a process to an ambient of group $\gamma$ : this is ensured by the constraint $\langle\varnothing,\{\gamma\}\rangle\langle u$. As in rules (In-I) and (OUT-I), we add to the environment scheme the premises $\alpha$ :amb and $\gamma$ : group only if $\alpha, \gamma$ are variables.

In rule (INPUT-I) the communication type scheme $t$ is equated with $\operatorname{com}(w)$ where $w$ is a fresh message variable, the input binder is annotated with $w$, and $x$ is removed from the environment. In fact $\Theta \downarrow x$ is defined as $\{y: W \mid y: W \in$ $\Theta \& y \neq x\}$. We add to the set of constraints the equality (if any) generated by combining the assumption $x: w$ with the environment scheme of the premise. In order to take into account the conditions $x \notin \Sigma$ and $x \notin(\mathrm{U}, \operatorname{com}(\mathrm{W}))$ of rule (INPUT) we add the constraint $x \notin\{w \mid w \in \Theta \downarrow x\} \cup\{u, t\}$.

Rule (Output-I) is similar but simpler: we equate the communication type scheme $t$ of the process scheme with $\operatorname{com}(W)$ where $W$ is the message type scheme of the output. We deal with the environment schemes and the constraints of the premises as in rule (CAP PREFIX-I).

In rule (AMB-I) the mobcom type scheme of the ambient is the mobcom type scheme of the enclosed process scheme and the mobcom type scheme of the whole process scheme is just $(u, t)$ with $u$ and $t$ fresh.

Rule (PAR-I) needs to ensure that the two process schemes have the same mobcom type scheme: this is achieved by adding to the constraint set the equality between the mobility variables and the communication type schemes of these process schemes.

Finally, in rule (GRP RES-I) we need to ensure that $g$ will not appear in the environment or in the process type: this is accomplished by adding the constraint $g \notin\{\xi \mid \xi \in \Theta \vee \xi \in V\}$.

The following properties of the inference rules can be easily proved by inspection of the rules themselves.

Proposition 3. Let $R \Longrightarrow\left\langle\Theta \vdash_{I} S: V \| \mathcal{C}\right\rangle$. Then:

1. $x: W \in \Theta$ implies $W \in\{w$, amb, group $\}$ for some $w$;

2. $V=(u, t)$ for some $u, t$;

3. $\mathcal{C}=\mathcal{C}_{=} \cup \mathcal{C}_{\leq} \cup \mathcal{C}_{\notin}$ where:

(a) $\mathcal{C}_{=}$is a set of equalities of the following forms (equalities symmetric of the listed ones are omitted):

$$
\begin{array}{ll}
\mathrm{amb}=\mathrm{amb} & \text { group }=\operatorname{group} \mathrm{amb}=\operatorname{group} \mathrm{amb}=\operatorname{cap}(u) \\
\operatorname{group}=\operatorname{cap}(u) & t=t^{\prime} \quad w=w^{\prime} \quad u=u^{\prime}
\end{array}
$$




$$
\begin{array}{llll}
t=\operatorname{com}(\mathrm{amb}) & t=\operatorname{com}(\operatorname{group}) & t=\operatorname{com}(w) & t=\operatorname{com}(\operatorname{cap}(u)) \\
w=\operatorname{amb} & w=\operatorname{group} & w=\operatorname{cap}(u) & \operatorname{cap}(u)=\operatorname{cap}\left(u^{\prime}\right)
\end{array}
$$

(b) $\mathcal{C}_{\leq}$is a set of subtyping judgments of the form $w \leq w^{\prime}$ and $\mathrm{U} \leq u$;

(c) $\mathcal{C}_{\notin}$ is a set of constraints of the form $\gamma \notin\left\{w_{1}, \ldots, w_{k}, u, t\right\}$ where $k \geq 0$.

We are now going to describe a procedure solve which, applied to a set of constraints, checks if it is solvable. If the set of constraints is solvable solve generates a particular solution of it, otherwise fails. The procedure makes use of three sub-procedures, executed in sequence: solve $=$, which solves the equality constraints; solve $\leq$, which solves the inequalities; and solve $\notin$, which checks the non-occurrence conditions. Any of the three may fail; in this case the set of constraints has no solution, the algorithm stops and the given term is not typable. If all succeed, then solve provides a solution.

The procedure solve $=$ applies the standard unification algorithm to $\mathcal{C}=$. By Proposition 3(3a), $\mathcal{C}=$ is a set of equations, therefore solve $=$ either fails or returns a substitution $\sigma=$ which is a most general unifier. It is easy to verify that $\sigma_{=}$ maps communication type variables to communication type schemes different from shh, message type variables to message type schemes and mobility type variables to mobility type variables.

If $\mathcal{C}=$ is solvable, then the main procedure solve applies the substitution $\sigma=$ to both sides of the subtyping judgments in $\mathcal{C}_{\leq}$, thus obtaining a set of subtyping judgments $\mathcal{C}_{\leq}^{1}$. Since the subtyping judgments in $\mathcal{C}_{\leq}$are of the forms $\mathrm{U} \leq u$ and $w \leq w^{\prime}$, then $\mathcal{C}_{<}^{1}$ contains only judgments of the forms $\mathrm{U} \leq u$ and $W \leq W^{\prime}$, where $W$ and $W^{\top}$ are either amb, or group, or capability type $\operatorname{schemes} \operatorname{cap}(u)$, or message type variables $w$.

The procedure solve $\leq$ starts by defining a substitution $\sigma_{W}$ from message type variables that occur in $\mathcal{C}_{\leq}^{1}$ to message type schemes. To this end we build the transitive closure of the relation $\leq$ in $\mathcal{C}_{\leq}^{1}$, i.e., we add $W_{1} \leq W_{3}$ to $\mathcal{C}_{\leq}^{1}$ whenever $W_{1} \leq W_{2}$ and $W_{2} \leq W_{3}$ are in it, for some $W_{2}$. Let $\mathcal{C}_{\leq}^{2}$ be the resulting set of inequalities.

Assume initially $\sigma_{W}$ as the trivial identity substitution and repeat the following steps (which transform both $\sigma_{W}$ and $\mathcal{C}_{\leq}^{2}$ ) until possible:

1. for all $w$ such that $a m b \leq w$ or $w \leq$ amb set $\sigma_{W}(w)=$ amb and replace $w$ with amb in $\mathcal{C}_{<}^{2}$;

2. for all $w$ such that group $\leq w$ or $w \leq$ group set $\sigma_{W}(w)=$ group and replace $w$ with group in $\mathcal{C}_{<}^{2}$;

3. for all $w$ such that $\operatorname{cap}\left(u^{\prime}\right) \leq w$ or $w \leq \operatorname{cap}\left(u^{\prime}\right)$ set $\sigma_{W}(w)=\operatorname{cap}(u)$, where $u$ is fresh, and replace $w$ with $\operatorname{cap}(u)$ in $\mathcal{C}_{\leq}^{2}$.

At the end, define $\sigma_{W}(w)=$ group for all remaining message type variables $w$ and replace them accordingly in $\mathcal{C}_{\leq}^{2}$. Let $\mathcal{C}_{\leq}^{3}$ be the so obtained set of constraints.

For instance, if $\mathcal{C}_{\leq}^{2}=\left\{w_{1} \leq w_{2}, w_{1} \leq\right.$ group $\}$, after the first iteration we get $\sigma_{W}=i d\left\{w_{1}:=\right.$ group $\}$ (where $i d$ is the identity substitution) and $\mathcal{C}_{\leq}^{2}=\{$ group $\leq$ $w_{2}$, group $\leq$ group $\}$ and after the second iteration $\sigma_{W}=i d\left\{w_{1}:=\right.$ group, $w_{2}:=$ 
group $\}$ and $\mathcal{C}_{\leq}^{2}=\mathcal{C}_{\leq}^{3}=\{$ group $\leq$ group $\}$. Note the need, in defining $\sigma_{W}$, to iterate the previous steps more than once.

If $\mathcal{C}_{<}^{3}$ is inconsistent (i.e., if it contains inequalities involving amb and group, or $\operatorname{amb}$ and $\operatorname{cap}(u)$, or $\operatorname{cap}(u)$ and group) then solve $\leq$ fails. Otherwise the nontrivial subtyping judgments in $\mathcal{C}_{<}^{3}$ are of the forms $\operatorname{cap}(u) \leq \operatorname{cap}\left(u^{\prime}\right)$ and $\mathrm{U} \leq u$. The procedure solve $\leq$ applies the following transformations to $\mathcal{C}_{\leq}^{3}$ :

- replace $\langle\mathscr{C}, \mathscr{E}\rangle \leq u$ and $\left\langle\left\langle\mathscr{C}^{\prime}, \mathscr{E}\right\rangle\right\rangle \leq u$ with $\left.\left\langle\mathscr{C} \cup \mathscr{C}^{\prime}, \mathscr{E} \cup \mathscr{E}\right\rangle\right\rangle \leq u$

- add $\mathrm{U} \leq u$ whenever $\operatorname{cap}\left(u^{\prime}\right) \leq \operatorname{cap}(u)$ and $\mathrm{U} \leq u^{\prime}$

until a fixed point $\mathcal{C}_{\leq}^{4}$ is reached. Now define for all mobility variables $u$ :

$$
\sigma_{U}(u)= \begin{cases}U & \text { if } \mathrm{U} \leq u \in \mathcal{C}_{\leq}^{4} \\ \langle\varnothing, \varnothing\rangle & \text { otherwise }\end{cases}
$$

Finally, the main procedure solve calls solve $\notin$, which checks the satisfiability of the constraints in $\mathcal{C}_{\notin}$. If there are $\gamma$ and $\xi$ such that the constraint $\gamma \notin \mathcal{I}$ is in $\mathcal{C}_{\notin}$, and $\xi \in \mathcal{I}$ and $\gamma \in \sigma_{U} \circ \sigma_{W} \circ \sigma_{=}(\xi)$, then the result of solve $\notin$ is failure; otherwise solve $\notin$ returns success.

For the output of solve to be a ground substitution, the remaining communication type variables must be replaced by types; then, only in the case solve $\notin$ returned success, define:

$$
\sigma_{T}(t)=\operatorname{shh}
$$

The output of the whole procedure solve is finally defined as

$$
\text { solve }(\mathcal{C})= \begin{cases}\sigma_{T} \circ \sigma_{U} \circ \sigma_{W} \circ \sigma_{=} & \text {if all substitutions are defined, } \\ \text { failure } & \text { otherwise. }\end{cases}
$$

The proof of the following proposition is straightforward:

Proposition 4. If the set of constraints $\mathcal{C}$ is solvable then solve $(\mathcal{C})$ is a solution of $\mathcal{C}$. Vice versa, if $\mathcal{C}$ is not solvable then solve $(\mathcal{C})$ fails.

In the rest of this section we will prove soundness and completeness of our inference algorithm. Crucial here is the treatment of free and bound variables. While the type assignment system considers processes modulo renaming of bound variables and names, the inference procedure cannot, since bound variables and names may appear in the set of constraints. For instance, we get

$$
(x) \text {. to } n: g \cdot \operatorname{co} g^{\prime} \text { with }\langle\{x\}, \varnothing\rangle \cdot 0 \Longrightarrow\left\langle\vdash_{I} S_{0}:(u, t) \| \mathcal{C}_{0}\right\rangle
$$

where

$$
S_{0}=(x: w) \cdot \text { to } n: g \text { with }\left(u^{\prime}, t^{\prime}\right) \cdot \operatorname{co} g^{\prime} \text { with }\langle\{x\}, \varnothing\rangle \cdot 0
$$

and

$$
\begin{aligned}
\mathcal{C}_{0}=\left\{\langle\{x\}, \varnothing \rrbracket\rangle u^{\prime \prime}, \operatorname{cap}\left(u^{\prime \prime}\right)=\operatorname{cap}\left(u^{\prime}\right),\langle\ell \varnothing,\{g\}\rangle \leq u, t=\operatorname{com}(w), w=\text { group },\right. \\
x \notin\{u, t\}\} .
\end{aligned}
$$


Note that $\mathcal{C}_{0}$ contains constraints involving the bound variable $x$. The substitution $\varsigma_{0}=\operatorname{solve}\left(\mathcal{C}_{0}\right)$ for the current variables is defined by $\varsigma_{0}\left(u^{\prime \prime}\right)=\varsigma_{0}\left(u^{\prime}\right)=$ $\|\{x\}, \varnothing\rangle, \varsigma_{0}(u)=\langle\varnothing,\{g\}\rangle, \varsigma_{0}(t)=\operatorname{com}($ group $), \varsigma_{0}(w)=$ group, $\varsigma_{0}\left(t^{\prime}\right)=$ shh. As will follow from Theorem [3, applying $\varsigma_{0}$ to the inferred process scheme $S_{0}$ gives a good process; however, to be able to apply $\varsigma_{0}$, we are forced to introduce an occurrence of $x$ in the scope of the binder, because $u^{\prime}$ must be replaced with $\langle\{x\}, \varnothing\rangle$. We conclude that:

when we apply a substitution to a process scheme in the scope of a binder, we need to allow capturing of group variables and names.

Therefore, following the 'nomenclature' of [16, we say that type variables are replaced by types.

As regards soundness, note that if the statement $M \Longrightarrow\left\langle\Theta \vdash_{I} M: W \| \mathcal{C}\right\rangle$ is derivable from the type inference rules and $\varsigma$ is a solution of $\mathcal{C}$, then $\varsigma(\Theta)$ may not be a good environment because the mobility types appearing in it might contain variables which either are assigned a type different from group or are not in its domain. To obtain a deducible typing judgment we therefore have to require that the enlarged environment $\varsigma(\Theta) \cup \Sigma$ is a good environment, where the domain of $\Sigma$ contains the missing group variables. The case of statements of the form $R \Longrightarrow\left\langle\Theta \vdash_{I} S: V \| \mathcal{C}\right\rangle$ is analogous.

\section{Theorem 3 (Soundness of Inference).}

1. If $M \Longrightarrow\left\langle\Theta \vdash_{I} M: W \| \mathcal{C}\right\rangle$ holds and $\varsigma$ is a solution of $\mathcal{C}$ such that $\varsigma(\Theta) \cup \Sigma$ is good, with $\Sigma=\{x$ : group $\mid \exists \xi .(\xi \in \Theta \vee \xi \in W) \wedge x \in \varsigma(\xi)\}$, then the typing $\varsigma(\Theta) \cup \Sigma \vdash \varsigma(M): \varsigma(W)$ is derivable.

2. If $R \Longrightarrow\left\langle\Theta \vdash_{I} S: V \| \mathcal{C}\right\rangle$ holds, then $|S|=R$; also, if $\varsigma$ is a solution of $\mathcal{C}$ such that $\varsigma(\Theta) \cup \Sigma$ is good, with $\Sigma=\{x$ : group $\mid \exists \xi .(\xi \in \Theta \vee \xi \in S \vee \xi \in V) \wedge x \in \varsigma(\xi)\}$, then the typing $\varsigma(\Theta) \cup \Sigma \vdash \varsigma(S): \varsigma(V)$ is derivable.

Proof. The proofs of (1) and (2) are respectively by induction on the derivations of $M \Longrightarrow\left\langle\Theta \vdash_{I} M: W \| \mathcal{C}\right\rangle$ and $R \Longrightarrow\left\langle\Theta \vdash_{I} S: V \| \mathcal{C}\right\rangle$. We only consider two representative cases.

Let the last rule applied be (CAP PrefIX-I):

$$
\frac{\chi \Longrightarrow\left\langle\Theta \vdash_{I} \chi: W \| \mathcal{C}\right\rangle \quad R \Longrightarrow\left\langle\Theta^{\prime} \vdash_{I} S:(u, t) \| \mathcal{C}^{\prime}\right\rangle}{\chi \cdot R \Longrightarrow\left\langle\Theta \triangleright \Theta^{\prime} \vdash_{I} \chi \cdot S:(u, t) \| \mathcal{C} \cup \mathcal{C}^{\prime} \cup\{W=\operatorname{cap}(u)\} \cup \Theta \triangleright \Theta^{\prime}\right\rangle}
$$

The theorem's first conclusion $|\chi \cdot S|=\chi \cdot R$ is immediate since $|\chi \cdot S|=\chi \cdot|S|$ and by induction $|S|=R$. As for the second and more important conclusion, first of all observe that a solution $\varsigma$ of the set of constraints $\mathcal{C} \cup \mathcal{C}^{\prime} \cup\{W=$ $\operatorname{cap}(u)\} \cup \Theta \diamond \Theta^{\prime}$ is also a solution of $\mathcal{C}, \mathcal{C}^{\prime}$, and $\Theta \diamond \Theta^{\prime}$. A first consequence is that by Proposition 2 one has $\varsigma\left(\Theta \triangleright \Theta^{\prime}\right)=\varsigma(\Theta) \cup \varsigma\left(\Theta^{\prime}\right)$. Now put

$$
\begin{aligned}
& \Sigma=\{x: \text { group } \mid \exists \xi \cdot(\xi \in \Theta \vee \xi \in W) \wedge x \in \varsigma(\xi)\} \\
& \Sigma^{\prime}=\left\{x \text { : group } \mid \exists \xi \cdot\left(\xi \in \Theta^{\prime} \vee \xi \in S \vee \xi \in(u, t)\right) \wedge x \in \varsigma(\xi)\right\} \\
& \Sigma^{\prime \prime}=\left\{x \text { group } \mid \exists \xi .\left(\xi \in\left(\Theta \triangleright \Theta^{\prime}\right) \vee \xi \in(\chi . S) \vee \xi \in(u, t)\right) \wedge x \in \varsigma(\xi)\right\} .
\end{aligned}
$$


The constraint $W=\operatorname{cap}(u)$ implies $\varsigma(W)=\varsigma(\operatorname{cap}(u))$ and then $\Sigma^{\prime \prime}=\Sigma \cup \Sigma^{\prime}$. If we assume that $\Sigma^{\prime \prime} \cup\left(\Theta \triangleright \Theta^{\prime}\right)$ is a good environment, then so are $\Sigma \cup \Theta$ and $\Sigma^{\prime} \cup \Theta^{\prime}$; then by (1) we get $\varsigma(\Theta) \cup \Sigma \vdash \varsigma(\chi): \varsigma(W)$, and by induction on (2) we get $\varsigma\left(\Theta^{\prime}\right) \cup \Sigma^{\prime} \vdash \varsigma(S): \varsigma((u, t))$.

By weakening (Lemma 4(3) ) we have $\varsigma\left(\Theta \triangleright \Theta^{\prime}\right) \cup \Sigma^{\prime \prime} \vdash \varsigma(\chi): \varsigma(W)$ and also $\varsigma\left(\Theta \triangleright \Theta^{\prime}\right) \cup \Sigma^{\prime \prime} \vdash \varsigma(S): \varsigma((u, t))$. Being $\varsigma(W)=\varsigma(\operatorname{cap}(u))$ we can conclude by applying the rule (CAP PREFIX).

Consider now the case where the last rule applied is (INPUT-I):

$$
\frac{R \Longrightarrow\left\langle\Theta \vdash_{I} S:(u, t) \| \mathcal{C}\right\rangle}{(x) . R \Longrightarrow\left\langle\Theta \downarrow x \vdash_{I}(x: w) . S:(u, t) \| \mathcal{C} \cup\{t=\operatorname{com}(w), x \notin \mathcal{I}\} \cup\{x: w\} \diamond \Theta\right\rangle}
$$

where $\mathcal{I}=\{w \mid w \in \Theta \downarrow x\} \cup\{u, t\}$. Since by hypothesis $\varsigma$ solves $x \notin \mathcal{I}$, we get $x \notin$ $\varsigma(\Theta \downarrow x)$ and $x \notin \varsigma((u, t))$. As in the previous case, by induction, by Proposition 2 and by Lemma 4(3) we get $\varsigma(\{x: w\} \triangleright \Theta) \cup \Sigma \vdash \varsigma(S): \varsigma((u, t))$, with $\Sigma$ properly defined following the type inference rule. By definition $\{x: w\} \triangleright \Theta=\{x: w\} \cup \Theta \downarrow x$ and the constraint $\{t=\operatorname{com}(w)\}$ implies $\varsigma(t)=\varsigma(\operatorname{com}(w))$. So we conclude by applying the rule (INPUT).

It is not surprising that the output of solve turns out to produce a derivable statement:

Corollary 2. If $R \Longrightarrow\left\langle\Theta \vdash_{I} S: V \| \mathcal{C}\right\rangle$ and $\varsigma=\operatorname{solve}(\mathcal{C})$, then $\varsigma(\Theta) \vdash \varsigma(S)$ : $\varsigma(V)$ is derivable.

Proof. By inspection of the type inference rules and of the solve procedure it is easy to check that if $x$ occurs in the range of solve $(\mathcal{C})$ then $R$ contains a co-move co $\gamma$ with $\mathrm{U}$ for some $\gamma$, $\mathrm{U}$ such that $x \in \mathrm{U}$. By rule (CO-I) the assumption $x$ : group is in $\Theta$; therefore $\{x$ : group $\mid \exists \xi$. $(\xi \in \Theta \vee \xi \in S \vee \xi \in V) \wedge x \in \varsigma(\xi)\}$ is a subset of $\Theta$, and we can conclude by Theorem 3

We can state and prove completeness as expected.

\section{Theorem 4 (Completeness of Inference).}

1. If $\Sigma \vdash M: \mathrm{W}$, then $M \Longrightarrow\left\langle\Theta \vdash_{I} M: W \| \mathcal{C}\right\rangle$ and there is a solution $\varsigma$ of $\mathcal{C}$ such that $\varsigma(\Theta) \subseteq \Sigma$ and $\varsigma(W)=\mathrm{W}$.

2. If $\Sigma \vdash P: \mathrm{V}$, then $|P| \Longrightarrow\left\langle\Theta \vdash_{I} S: V \| \mathcal{C}\right\rangle$ and there is a solution $\varsigma$ of $\mathcal{C}$ such that $\varsigma(\Theta) \subseteq \Sigma$ and $\varsigma(S)=P$ and $\varsigma(V)=\mathrm{V}$.

Proof. The proofs of (11) and (2) are respectively by induction on the derivations of $\Sigma \vdash M: \mathrm{W}$ and $\Sigma \vdash P: \mathrm{V}$. We only consider some representative cases.

If the last rule applied is (CAP PREFIX):

$$
\frac{\Sigma \vdash \chi: \operatorname{cap}(\mathrm{U}) \quad \Sigma \vdash P:(\mathrm{U}, \mathrm{T})}{\Sigma \vdash \chi \cdot P:(\mathrm{U}, \mathrm{T})}
$$

then 
- by (11), $\chi \Longrightarrow\left\langle\Theta \vdash_{I} \chi: W \| \mathcal{C}\right\rangle$ and there is a solution $\varsigma_{1}$ of $\mathcal{C}$ such that $\varsigma_{1}(\Theta) \subseteq \Sigma$ and $\varsigma_{1}(W)=\operatorname{cap}(\mathrm{U})$

- by induction on (2), $|P| \Longrightarrow\left\langle\Theta^{\prime} \vdash_{I} S:(u, t) \| \mathcal{C}^{\prime}\right\rangle$ and there is a solution $\varsigma_{2}$ of $\mathcal{C}^{\prime}$ such that $\varsigma_{2}\left(\Theta^{\prime}\right) \subseteq \Sigma$ and $\varsigma_{2}(S)=P$ and $\varsigma_{2}((u, t))=(\mathrm{U}, \mathrm{T})$.

By rule (CAP PREFIX-I) we get:

$$
\frac{\chi \Longrightarrow\left\langle\Theta \vdash_{I} \chi: W \| \mathcal{C}\right\rangle \quad|P| \Longrightarrow\left\langle\Theta^{\prime} \vdash_{I} S:(u, t) \| \mathcal{C}^{\prime}\right\rangle}{\chi \cdot|P| \Longrightarrow\left\langle\Theta \triangleright \Theta^{\prime} \vdash_{I} \chi \cdot S:(u, t) \| \mathcal{C} \cup \mathcal{C}^{\prime} \cup\{W=\operatorname{cap}(u)\} \cup \Theta \triangleright \Theta^{\prime}\right\rangle}
$$

We can assume that the sets of type variables which occur free in $\mathcal{C}$ and $\mathcal{C}^{\prime}$ are disjoint and define for all type variables $\xi$ :

$$
\varsigma(\xi)= \begin{cases}\varsigma_{1}(\xi) & \text { if } \xi \in \mathcal{C} \\ \varsigma_{2}(\xi) & \text { otherwise }\end{cases}
$$

By construction $\varsigma$ is a solution of both $\mathcal{C}$ and $\mathcal{C}^{\prime}$. Moreover, since $\varsigma(W)=\operatorname{cap}(\mathrm{U})$ and $\varsigma((u, t))=(\mathrm{U}, \mathrm{T})$, the substitution $\varsigma$ solves the constraint $W=\operatorname{cap}(u)$. Lastly, $\varsigma(\Theta) \subseteq \Sigma$ and $\varsigma\left(\Theta^{\prime}\right) \subseteq \Sigma$ imply that for all term variables $x$ if $x: w \in \Theta$ and $x: w^{\prime} \in \Theta^{\prime}$ then $\varsigma(w)=\varsigma\left(w^{\prime}\right)$, i.e., $\varsigma$ is also a solution of $\Theta \triangleright \Theta^{\prime}$. By Proposition 2 this implies $\varsigma\left(\Theta \triangleright \Theta^{\prime}\right)=\varsigma(\Theta) \cup \varsigma\left(\Theta^{\prime}\right)$ and then we get $\varsigma\left(\Theta \triangleright \Theta^{\prime}\right) \subseteq \Sigma$.

If the last rule applied is (INPUT):

$$
\frac{\Sigma, x: \mathrm{W} \vdash P:(\mathrm{U}, \operatorname{com}(\mathrm{W})) \quad x \notin \Sigma \quad x \notin(\mathrm{U}, \operatorname{com}(\mathrm{W}))}{\Sigma \vdash(x: \mathrm{W}) \cdot P:(\mathrm{U}, \operatorname{com}(\mathrm{W}))}
$$

then by induction $|P| \Longrightarrow\left\langle\Theta \vdash_{I} S:(u, t) \| \mathcal{C}\right\rangle$ and there is a solution $\varsigma$ of $\mathcal{C}$ such that $\varsigma(\Theta) \subseteq \Sigma, x: \mathrm{W}$ and $\varsigma(S)=P$ and $\varsigma((u, t))=(\mathrm{U}, \operatorname{com}(\mathrm{W}))$. Since $\varsigma(t)=\operatorname{com}(\mathrm{W})$ and $\varsigma(\Theta) \subseteq \Sigma, x$ : W, the substitution $\varsigma$ is also a solution of $\{t=$ $\operatorname{com}(w)\} \cup\{x: w\} \diamond \Theta$. The condition $x \notin \Sigma$ implies $x \notin \varsigma(\Theta \downarrow x)$; moreover $x \notin$ $(\mathrm{U}, \operatorname{com}(\mathrm{W}))$. Therefore $\varsigma$ satisfies also the constraint $x \notin\{w \mid w \in \Theta \downarrow x\} \cup\{u, t\}$.

\section{Conclusions and Related Work}

We have introduced a variant of the Calculus of Mobile Ambients (MA) that combines ambient and process mobility and allows the expression of flexible policies for controlling process activities. The calculus exploits co-move actions and runtime type checking to require the agreement between a moving process and the target ambient (similar mechanisms could also be used for ambient movements, but we have omitted them for the sake of simplicity). Policies can dynamically change due to further co-moves being added to an ambient, either by means of process movements or indirectly through communication. The operational semantics and the type assignment system ensure that an incoming process conforms to the policy of the target ambient. The compliance with ambient policies can be checked locally and requires no global assumption. We 
have defined a sound and complete type inference algorithm and illustrated a few applications of our framework to examples.

As future work, we are considering ways of increasing the expressive power of our type system so as to be able to express stronger properties. For instance, incoming processes are only checked against mobility capabilities. No check about their input/output behaviour is currently done, except that their communication type must comply with the communication type of the entered ambient. Instead, one could need a more strict control as, for example, in the scenario of Section 2 where reader processes entering a journal could be allowed only to read (i.e., input) papers, not to write (i.e., output) them. This would require distinguishing between input and output, as is usually done in calculi with channel-based communication (see, e.g., 35). One could also obtain more informative policies by asking that the operation of ambient creation be subject to authorization. This would be especially significant when creating ambients of known groups, in which case, in general, one may expect they behave in a controlled way.

\section{Related Work}

Modelling wide-area distributed systems requires that the space of locations and the mobility in such space are taken into account as new dimensions of computing. Most foundational languages proposed in the literature model this space either as an evolving graph of fully connected locations, like the $\mathrm{D} \pi$ [27] and the language KLAIM [17, or as an evolving forest of trees of locations, like MA [12 and its variants. Some recent proposals explicitly handling the underlying network topology are tKLAim [18] and $\mathrm{D} \pi_{F}$ [20. An interesting core model generalizing many of the available calculi and languages has been developed within the Mikado project [5].

Many variants of MA have been defined: for a survey see [21. A crucial choice in all these calculi is the form of interaction between processes in different ambients. In the original calculus [12] interaction is only local to an ambient, and for processes in different ambients to communicate, at least one of the ambients' boundaries has to be dissolved by means of the open capability. This approach has also been used in [12|29|6|31|1]. However, the open capability has been considered by many researchers as potentially dangerous, because it could be inadvertently or maliciously used to destroy an ambient's individuality (by dissolving its boundary). Therefore, several variants of MA have been proposed which either are equipped with additional constructs for controlling the execution of open, like the co-moves of Safe Ambients 29 (used with modifications also in $6[31|32| 8]$ ), or replace it with other interaction-enabling mechanisms: among them, we mention communication between nested ambients in Boxed Ambients [7/32 [8] and in Seal calculus [13], and process (objective) mobility in [15]14. To ensure and enforce behavioural properties, in particular those concerning resource access, communication, mobility and security, ambient calculi are usually typed [10/29/116/1/7/31/32/8/30.

The calculus presented in this paper is derived from the variant of $\mathbf{M}^{3}$ presented in 14. However, the two calculi use different authorization mechanisms. 
In 14, rights to cross or enter an ambient are recorded as passive components (i.e., multisets of rights attached to the ambient); here, authorization relies on co-moves and rights, which allow more flexible policies. Also the mechanisms used to pass permits are different: in [14 specific primitives are used to add permissions to the multisets attached to ambients; the mechanism used in this paper, on the contrary, has been somehow inspired by [24], where policies to access network resources can dynamically change due to communication of permissions.

Before the present calculus, only the calculi of [26|28|6|24|4|23], to our knowledge, considered type information local to computational environments, while in the other proposals there is a global environment containing all typing assumptions. In [26]24|28|23] local type information is sufficient because processes are dynamically checked whenever they migrate, which prevents processes not complying with the policies of a locality to get in. This is similar to our approach, though their computational environments are not hierarchically structured. To reduce the amount of dynamic controls, in 28 a relation of trust among nodes is exploited; thus, a process coming from a trusted node is never dynamically type-checked. In 23] each location comes equipped with a membrane that controls access by type-checking the incoming processes. The presence of the open capability requires in [6] a careful updating of the local type information of ambients when they migrate. The aim of types in 4 is dual to ours: the type system ensures a liberal but safe communication policy, so that ambient movements are only allowed when this does not break the soundness of data exchanges. Also, the calculus of 4 is a variant of Boxed Ambients and therefore communication may cross one ambient boundary.

Dependent types have been widely used in the framework of the calculus $\mathrm{D} \pi$ (see, e.g., [25]) to restrict capabilities of processes launched by incoming code. To our knowledge, the type system of [30, where types directly depend on ambient variables, is the only with dependent types for variants of MA. Our type system is simpler but less precise than the one of [30] in the specification of ambient behaviours, since in our approach all ambients of the same group share the same "passive" behaviour.

The first type inference algorithm for MA was presented in [36]. Other algorithms for variants of typed ambient calculi can be found in [2] and [15]. The main challenge in the design of the inference algorithm presented in this paper has been the handling of dependent types, an issue not addressed by any of the above.

\section{References}

1. T. Amtoft, A. J. Kfoury, and S. M. Pericas-Geertsen. What are PolymorphicallyTyped Ambients? In D. Sands, editor, Proc. of ESOP'01, volume 2028 of LNCS, pages 206-220. Springer, 2001.

2. F. Barbanera, M. Dezani-Ciancaglini, I. Salvo, and V. Sassone. A type inference algorithm for secure ambients. In M. Lenisa and M. Miculan, editors, Proc. of TOSCA'01, volume 62 of ENTCS. Elsevier Science, 2002. 
3. L. Bettini, V. Bono, R. D. Nicola, G. Ferrari, D. Gorla, M. Loreti, E. Moggi, R. Pugliese, E. Tuosto, and B. Venneri. The KLAIM Project: Theory and Practice. In C. Priami, editor, Global Computing: Programming Environments, Languages, Security and Analysis of Systems, volume 2874 of LNCS, pages 88-151. Springer, 2003.

4. E. Bonelli, A. Compagnoni, M. Dezani-Ciancaglini, and P. Garralda. Boxed Ambients with Communication Interfaces. In V. Fiala, Jiríand Koubek and K. Jan, editors, Proc. of MFCS '04, volume 3153 of $L N C S$, pages 119-148. Springer, 2004.

5. G. Boudol. A Parametric Model of Migration and Mobility, Release 1. Mikado Deliverable D1.2.1, available at http://mikado.di.fc.ul.pt/repository/D1.2.1.pdf, 2003.

6. M. Bugliesi and G. Castagna. Behavioral Typing for Safe Ambients. Computer Languages, 28(1):61 - 99, 2002.

7. M. Bugliesi, G. Castagna, and S. Crafa. Access Control for Mobile Agents: The Calculus of Boxed Ambients. ACM Transactions on Programming Languages and Systems, 26(1):57-124, 2004.

8. M. Bugliesi, S. Crafa, M. Merro, and V. Sassone. Communication and Mobility Control in Boxed Ambients. Information and Computation, 202(1): 39-86, 2005.

9. L. Cardelli. Abstractions for Mobile Computation. In J. Vitek and C. Jensen, editors, Secure Internet Programming: Security Issues for Mobile and Distributed Objects, volume 1603 of LNCS, pages 51-94. Springer, 1999.

10. L. Cardelli, G. Ghelli, and A. D. Gordon. Mobility Types for Mobile Ambients. In J. Wiedermann, P. van Emde Boas, and M. Nielsen, editors, Proc. of ICALP'99, volume 1644 of $L N C S$, pages 230-239. Springer, 1999.

11. L. Cardelli, G. Ghelli, and A. D. Gordon. Types for the Ambient Calculus. Information and Computation, 177(2):160-194, 2002.

12. L. Cardelli and A. D. Gordon. Mobile Ambients. Theoretical Computer Science, 240(1):177-213, 2000. Special Issue on Coordination, Daniel Le Métayer Editor.

13. G. Castagna, J. Vitek, and F. Z. Nardelli. The Seal Calculus. Information and Computation, 201(1):1-54, 2005.

14. M. Coppo, M. Dezani-Ciancaglini, E. Giovannetti, and R. Pugliese. Dynamic and Local Typing for Mobile Ambients. In Proc. of TCS'04, pages 583-596. Kluwer, 2004.

15. M. Coppo, M. Dezani-Ciancaglini, E. Giovannetti, and I. Salvo. M3: Mobility Types for Mobile Processes in Mobile Ambients. In J. Harland, editor, Proc. of CATS'03, volume 78 of ENTCS. Elsevier, 2003.

16. H. B. Curry and R. Feys. Combinatory Logic, volume I of Studies in Logic and the Foundations of Mathematics. North-Holland, Amsterdam, 1958.

17. R. De Nicola, G. Ferrari, and R. Pugliese. Klaim: a Kernel Language for Agents Interaction and Mobility. IEEE Transactions on Software Engineering, 24(5):315330, 1998.

18. R. De Nicola, D. Gorla, and R. Pugliese. Basic observables for a calculus for global computing. In L. Caires, G. F. Italiano, L. Monteiro, C. Palamidessi, and M. Yung, editors, Proc. of ICALP'05, volume 3580 of LNCS, pages 1226-1238. Springer, 2005.

19. G. Ferrari, E. Moggi, and R. Pugliese. Guardians for Ambient-based Monitoring. In V. Sassone, editor, Proc. of F-WAN, volume 66 of ENTCS. Elsevier, 2002.

20. A. Francalanza and M. Hennessy. A theory of system behaviour in the presence of node and link failures. In M. Abadi and L. de Alfaro, editors, Proc. of CONCUR'05, volume 3653 of $L N C S$, pages 368-382. Springer, 2005. 
21. E. Giovannetti. Ambient Calculi with Types: a Tutorial. In C. Priami, editor, Global Computing - Programming Environments, Languages, Security and Analysis of Systems, volume 2874 of LNCS, pages 151-191. Springer, 2003.

22. H. Goguen. Typed Operational Semantics. In M. Dezani-Ciancaglini and G. Plotkin, editors, Proc. of TLCA'95, volume 902 of LNCS, pages 186-200. Springer, 1995.

23. D. Gorla, M. Hennessy, and V. Sassone. Security Policies as Membranes in Systems for Global Computing. In J. Rathke, editor, Proc. of FGUC'04, ENTCS. Elsevier, 2004.

24. D. Gorla and R. Pugliese. Resource Acces and Mobility Control with Dynamic Privileges Acquisition. In J. Parrow, editor, Proc. of ICALP'03, volume 2719 of LNCS, pages 119-132. Springer, 2003.

25. M. Hennessy, J. Rathke, and N. Yoshida. SafeDpi: A language for controlling mobile code (extended abstract). In I. Walukiewicz, editor, Proc. of FOSSACS'04, volume 2987 of LNCS, pages 241-256, 2004. Extended and revised version to appear in Acta Informatica.

26. M. Hennessy and J. Riely. Type-Safe Execution of Mobile Agents in Anonymous Networks. In J. Vitek and C. Jensen, editors, Secure Internet Programming: Security Issues for Distributed and Mobile Objects, number 1603 in LNCS, pages 95-115. Springer, 1999.

27. M. Hennessy and J. Riely. Resource Access Control in Systems of Mobile Agents. Information and Computation, 173:82-120, 2002.

28. M. Hennessy and J. Riely. Trust and Partial Typing in Open Systems of Mobile Agents. Journal of Automated Reasoning, 31(3-4):335-370, 2003.

29. F. Levi and D. Sangiorgi. Controlling Interference in Ambients. Transactions on Programming Languages and Systems, 25(1):1-69, 2003.

30. C. Lhoussaine and V. Sassone. A Dependently Typed Ambient Calculus. In D. Schmidt, editor, Proc. of ESOP'04, volume 2986 of LNCS, pages 171-187. Springer, 2004.

31. M. Merro and M. Hennessy. Bisimulation Congruences in Safe Ambients. In N. D. Jones and X. Leroy, editors, Proc. of POPL'02, pages 71-80, New York, 2002. ACM Press.

32. M. Merro and V. Sassone. Typing and Subtyping Mobility in Boxed Ambients. In L. Brim, P. Jancar, M. Kretinsky, and A. Kucera, editors, Proc. of CONCUR'02, volume 2421 of $L N C S$, pages 304-320. Springer, 2002.

33. G. C. Necula. Proof-Carrying Code. In N. D. Jones, editor, Proc. of POPL'97, pages 106-119. ACM Press, 1997.

34. B. C. Pierce. Types and Programming Languages. MIT Press, 2002.

35. B. C. Pierce and D. Sangiorgi. Typing and Subtyping for Mobile Processes. Mathematical Structures in Computer Science, 6(5):409-454, 1996. An extract appeared in Proc. of LICS '93: 376-385.

36. P. Zimmer. Subtyping and typing algorithms for mobile ambients. In J. Tiuryn, editor, Proc. of FOSSACS'00, volume 1784 of $L N C S$, pages 375-390. Springer, 2000. 